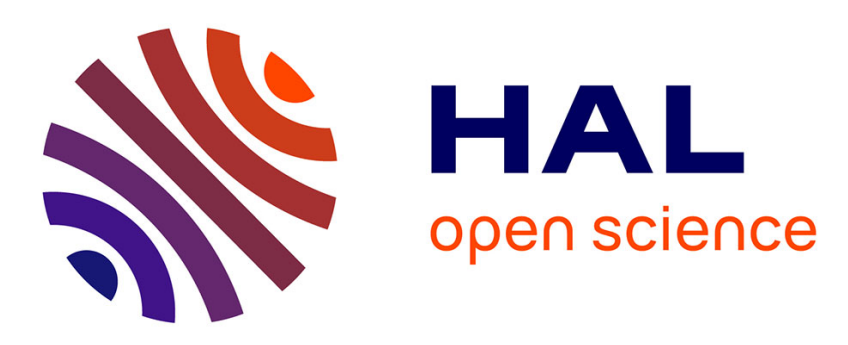

\title{
Spectral element discretization of the vorticity, velocity and pressure formulation of the Navier-Stokes problem
}

Karima Amoura, Mustapha Azaïez, Christine Bernardi, Nejmeddinen Chorfi, Samira Saadi

\section{- To cite this version:}

Karima Amoura, Mustapha Azaïez, Christine Bernardi, Nejmeddinen Chorfi, Samira Saadi. Spectral element discretization of the vorticity, velocity and pressure formulation of the Navier-Stokes problem. 2007. hal-00139603

\section{HAL Id: hal-00139603 https://hal.science/hal-00139603}

Preprint submitted on 2 Apr 2007

HAL is a multi-disciplinary open access archive for the deposit and dissemination of scientific research documents, whether they are published or not. The documents may come from teaching and research institutions in France or abroad, or from public or private research centers.
L'archive ouverte pluridisciplinaire HAL, est destinée au dépôt et à la diffusion de documents scientifiques de niveau recherche, publiés ou non, émanant des établissements d'enseignement et de recherche français ou étrangers, des laboratoires publics ou privés. 


\title{
Spectral element discretization \\ of the vorticity, velocity and pressure formulation of the Navier-Stokes problem
}

\author{
by K. Amoura ${ }^{1}$, M. Azaïez ${ }^{2}$, C. Bernardi ${ }^{3}$, N. Chorfi ${ }^{4}$ and S. Saadi ${ }^{1}$
}

\begin{abstract}
The two-dimensional Navier-Stokes equations, when provided with non standard boundary conditions which involve the normal component of the velocity and the vorticity, admit a variational formulation with three independent unknowns, the vorticity, the velocity and the pressure. We propose a discretization of this problem by spectral element methods. A detailed numerical analysis leads to optimal error estimates for the three unknowns and numerical experiments confirm the interest of the discretization.
\end{abstract}

Résumé: Les équations de Navier-Stokes bidimensionnelles munies de conditions aux limites non usuelles portant sur la composante normale de la vitesse et le tourbillon, admettent une formulation variationnelle qui comporte trois inconnues indépendantes: le tourbillon, la vitesse et la pression. Nous proposons une discrétisation de ce problème par une méthode d'éléments spectraux. Une analyse numérique détaillée permet d'établir des majorations d'erreur optimales pour les trois inconnues et des expériences numériques confirment l'intérêt de la discrétisation.

1 Université Badji-Mokhtar, Faculté des Sciences, Département de Mathématiques, B.P. 12, 23000 Annaba, Algérie. e-mail addresses: amouradz@yahoo.fr (K. Amoura), signor_2000@yahoo.fr (S. Saadi)

2 Laboratoire TREFLE (UMR C.N.R.S. 8508), Site E.N.S.C.P.B., 16 avenue Pey Berland, 33607 Pessac Cedex, France. e-mail address: azaiez@enscpb.fr (M. Azaïez)

3 Laboratoire Jacques-Louis Lions, C.N.R.S. \& Université Pierre et Marie Curie, B.C. 187, 4 place Jussieu, 75252 Paris Cedex 05, France. e-mail address: bernardi@ann.jussieu.fr (C. Bernardi)

4 Département de Mathématiques, Faculté des Sciences de Tunis, Campus Universitaire, 1060 Tunis, Tunisie. e-mail address: nejmeddine.chorfi@fst.rnu.tn (N. Chorfi) 



\section{Introduction.}

Let $\Omega$ be a bounded connected domain in $\mathbb{R}^{2}$. We introduce the unit outward normal vector $\boldsymbol{n}$ to $\Omega$ on $\partial \Omega$ and we consider the nonlinear problem

$$
\begin{cases}\nu \operatorname{curl} \omega+\omega \times \boldsymbol{u}+\operatorname{grad} p=\boldsymbol{f} & \text { in } \Omega, \\ \operatorname{div} \boldsymbol{u}=0 & \text { in } \Omega, \\ \omega=\operatorname{curl} \boldsymbol{u} & \text { in } \Omega, \\ \boldsymbol{u} \cdot \boldsymbol{n}=0 & \text { on } \partial \Omega, \\ \omega=0 & \text { on } \partial \Omega .\end{cases}
$$

Indeed it is readily checked that this system is equivalent to the full Navier-Stokes equations when provided with boundary conditions on the normal component of the velocity and the vorticity. Such conditions appear for a large number of flows, for instance in the case of a fluid on both sides of a membrane or for the well-known Green-Taylor flow, see [16].

In system (1.1), the unknowns are the vorticity of the fluid $\omega$, its velocity $\boldsymbol{u}$ and its pressure $p$. This formulation with three unknowns was first proposed in [11] and [17] (see also [12] and [1]) and seems the best appropriate for handling the type of boundary conditions we are interesred in, both for the Stokes and Navier-Stokes problems. We have decided to treat only the case of a two-dimensional domain: Indeed, the variational spaces are rather different in dimension 2 (where the vorticity is a scalar function) and in dimension 3 (where the vorticity is a vector field). Moreover, the existence of a solution in the three-dimensional case is, up to our knowledge, only proved for a smooth domain $\Omega$ when the viscosity is large enough, see [4]. So we first check the existence of a solution and its stability in the case of a possibly multiply-connected bidimensional domain.

We are interested in the spectral element discretization of system (1.1). The numerical analysis of discretizations of the Stokes problem relying on this formulation has first been performed for finite element methods, see [17] and the references therein. It has recently been extended to the case of spectral methods in [5] and of spectral element methods [2]. We also refer to [4] for a first work concerning the discretization of the Navier-Stokes equations (1.1) by spectral methods. The main idea of this paper is to extend these results to the case of spectral element methods.

We first describe the discrete problem and prove that it admits at least a solution. Next, relying on the arguments presented in [2], we perform its numerical analysis. By using the theory introduced in [8], we prove optimal error estimates for the three unknowns. It can be noted that this is a special property of the formulation that we use, since the approximation of the pressure for other formulations of the Stokes or Navier-Stokes problem is most often not optimal (see $[7, \S 24-26]$ ).

We describe the Newton type iterative algorithm that is used to solve the nonlinear discrete problem. Relying once more on the arguments in [8], we check its convergence. We also describe a possible algorithm for exhibiting an appropriate initial guess in order 
to initiate the Newton's method. We conclude with some numerical experiments which confirm the optimality of the discretization and justify the choice of this formulation.

An outline of the paper is as follows.

- In Section 2, we write the variational formulation of system (1.1) and recall the existence of a solution.

- Section 3 is devoted to the description of the spectral element discrete problem. We also prove the existence of a solution.

- Optimal error estimates are derived in Section 4.

- In Section 5, we present the iterative algorithm that is used for solving the discrete problem and prove its convergence.

- Some numerical experiments are presented in Section 6. 


\section{The velocity, vorticity and pressure formulation.}

In order to write the variational formulation of problem (1.1) and for the sake of precision, we first recall the definition of the scalar and vector curl operators in dimension 2: For any vector field $\boldsymbol{v}=\left(v_{x}, v_{y}\right)$ and any scalar function $\varphi$,

$$
\operatorname{curl} \boldsymbol{v}=\partial_{x} v_{y}-\partial_{y} v_{x}, \quad \operatorname{curl} \varphi=\left(\begin{array}{c}
\partial_{y} \varphi \\
-\partial_{x} \varphi
\end{array}\right)
$$

where all derivatives in the previous line are taken in the distribution sense. We also recall that, for any vector field $\boldsymbol{v}=\left(v_{x}, v_{y}\right)$ and any scalar function $\varphi$, the product $\varphi \times \boldsymbol{v}$ means the vector with components $\varphi v_{y}$ and $-\varphi v_{x}$.

We note that the boundary conditions in this problem are not sufficient to enforce the uniqueness of the solution in the case of multiply-connected domains even for the Stokes problem, see $[2, \S 2]$ for more details. We need the following notation.

Notation 2.1. Let $\Sigma_{j}, 1 \leq j \leq J$, be connected open curves, called "cuts", such that:

(i) Each $\Sigma_{j}$ is an open part of a smooth curve,

(ii) Each $\Sigma_{j}, 1 \leq j \leq J$, is contained in $\Omega$ and its two endpoints belong to two different connected components of $\partial \Omega$,

(iii) The intersection of $\Sigma_{j}$ and $\Sigma_{j^{\prime}}, 1 \leq j<j^{\prime} \leq J$, is empty,

(iv) The open set $\Omega^{\circ}=\Omega \backslash \cup_{j=1}^{J} \Sigma_{j}$ is simply-connected.

The existence of such $\Sigma_{j}$ is clear. We make the further assumption that the domain $\Omega^{\circ}$ is pseudo-Lipschitz, in the sense that, for each point $\boldsymbol{x}$ of $\partial \Omega^{\circ}$, the intersection of $\Omega^{\circ}$ with a smooth neighbourhood of $\boldsymbol{x}$ has one or two connected components and each of them has a Lipschitz-continuous boundary (we refer to [3, $\S 3 . \mathrm{a}]$ for a more precise definition). Then, the further conditions read

$$
\langle\boldsymbol{u} \cdot \boldsymbol{n}, 1\rangle_{\Sigma_{j}}=0, \quad 1 \leq j \leq J
$$

where $\langle\cdot, \cdot\rangle_{\Sigma_{j}}$ stands for the duality pairing between $H^{-\frac{1}{2}}\left(\Sigma_{j}\right)$ and $H^{\frac{1}{2}}\left(\Sigma_{j}\right)$.

We consider the standard spaces $L^{p}(\Omega), 1 \leq p \leq+\infty$, and also the full scale of Sobolev spaces $H^{s}(\Omega)$ and $H_{0}^{s}(\Omega), s \geq 0$. We introduce the domain $H(\operatorname{div}, \Omega)$ of the divergence operator, namely

$$
H(\operatorname{div}, \Omega)=\left\{\boldsymbol{v} \in L^{2}(\Omega)^{2} ; \operatorname{div} \boldsymbol{v} \in L^{2}(\Omega)\right\} .
$$

Since the normal trace operator: $\boldsymbol{v} \mapsto \boldsymbol{v} \cdot \boldsymbol{n}$ can be defined from $H(\operatorname{div}, \Omega)$ into $H^{-\frac{1}{2}}(\partial \Omega)$, see [13, Chap. I, Thm 2.5], we also consider its kernel

$$
H_{0}(\operatorname{div}, \Omega)=\{\boldsymbol{v} \in H(\operatorname{div}, \Omega) ; \boldsymbol{v} \cdot \boldsymbol{n}=0 \text { on } \partial \Omega\} .
$$

Finally, let $L_{0}^{2}(\Omega)$ stand for the space of functions in $L^{2}(\Omega)$ with a null integral on $\Omega$.

In view of conditions $(2.2)$ and according to $[6, \S 2.5]$, we introduce the space

$$
\mathbb{D}(\Omega)=\left\{\boldsymbol{v} \in H_{0}(\operatorname{div}, \Omega) ;\langle\boldsymbol{v} \cdot \boldsymbol{n}, 1\rangle_{\Sigma_{j}}=0,1 \leq j \leq J\right\} .
$$


We now consider the variational problem

Find $(\omega, \boldsymbol{u}, p)$ in $H_{0}^{1}(\Omega) \times \mathbb{D}(\Omega) \times L_{0}^{2}(\Omega)$ such that

$$
\begin{aligned}
& \forall \boldsymbol{v} \in \mathbb{D}(\Omega), \quad a(\omega, \boldsymbol{u} ; \boldsymbol{v})+K(\omega, \boldsymbol{u} ; \boldsymbol{v})+b(\boldsymbol{v}, p)=\langle\boldsymbol{f}, \boldsymbol{v}\rangle, \\
& \forall q \in L_{0}^{2}(\Omega), \quad b(\boldsymbol{u}, q)=0, \\
& \forall \varphi \in H_{0}^{1}(\Omega), \quad c(\omega, \boldsymbol{u} ; \varphi)=0,
\end{aligned}
$$

where $\langle\cdot, \cdot\rangle$ denotes the duality pairing between $H_{0}(\operatorname{div}, \Omega)$ and its dual space. The bilinear forms $a(\cdot, \cdot ; \cdot), b(\cdot, \cdot)$ and $c(\cdot, \cdot ; \cdot)$ are defined by

$$
\begin{array}{r}
a(\omega, \boldsymbol{u} ; \boldsymbol{v})=\nu \int_{\Omega}(\operatorname{curl} \omega)(\boldsymbol{x}) \cdot \boldsymbol{v}(\boldsymbol{x}) d \boldsymbol{x}, \quad b(\boldsymbol{v}, q)=-\int_{\Omega}(\operatorname{div} \boldsymbol{v})(\boldsymbol{x}) q(\boldsymbol{x}) d \boldsymbol{x}, \\
c(\omega, \boldsymbol{u} ; \varphi)=\int_{\Omega} \omega(\boldsymbol{x}) \varphi(\boldsymbol{x}) d \boldsymbol{x}-\int_{\Omega} \boldsymbol{u}(\boldsymbol{x}) \cdot(\operatorname{curl} \varphi)(\boldsymbol{x}) d \boldsymbol{x} .
\end{array}
$$

The trilinear form $K(\cdot, \cdot ; \cdot)$ is given by

$$
K(\omega, \boldsymbol{u} ; \boldsymbol{v})=\int_{\Omega}(\omega \times \boldsymbol{u})(\boldsymbol{x}) \cdot \boldsymbol{v}(\boldsymbol{x}) d \boldsymbol{x} .
$$

As a consequence of the density of the space of infinitely differentiable functions with a compact support in $\Omega$ in $H_{0}(\operatorname{div}, \Omega)$ and $H_{0}^{1}(\Omega)$, see [13, Chap. I, §2], we derive the following statement. It involves the solutions $q_{j}^{t}, 1 \leq j \leq J$, of the problem (see [3, Prop. 3.14] for more details on these functions)

$$
\begin{cases}-\Delta q_{j}^{t}=0 & \text { in } \Omega^{\circ}, \\ \partial_{n} q_{j}^{t}=0 & \text { on } \partial \Omega, \\ {\left[q_{j}^{t}\right]_{j^{\prime}}=\text { constant, }} & 1 \leq j^{\prime} \leq J, \\ {\left[\partial_{n} q_{j}^{t}\right]_{j^{\prime}}=0,} & 1 \leq j^{\prime} \leq J, \\ \left\langle\partial_{n} q_{j}^{t}, 1\right\rangle_{\Sigma_{j^{\prime}}}=\delta_{j j^{\prime}}, & 1 \leq j^{\prime} \leq J,\end{cases}
$$

where $[\cdot]_{j^{\prime}}$ denotes the jump through $\Sigma_{j^{\prime}}$ (making its sign precise is not needed in what follows). Note that each $\widetilde{\operatorname{grad}} q_{j}^{t}$ belongs to $H_{0}(\operatorname{div}, \Omega)$, where grad stands for the gradient defined in the distribution sense on $\Omega^{\circ}$, and that $H_{0}(\operatorname{div}, \Omega)$ is the direct sum of $\mathbb{D}(\Omega)$ and of the space spanned by the $\widetilde{\operatorname{grad}} q_{j}^{t}, 1 \leq j \leq J$.

Proposition 2.2. For any data $\boldsymbol{f}$ in the dual space of $H_{0}(\operatorname{div}, \Omega)$ satisfying

$$
\left\langle\boldsymbol{f}, \widetilde{\operatorname{grad}} q_{j}^{t}\right\rangle=0, \quad 1 \leq j \leq J
$$

problems (1.1) - (2.2) and (2.6) are equivalent, in the sense that any triple $(\omega, \boldsymbol{u}, p)$ in $H^{1}(\Omega) \times H(\operatorname{div}, \Omega) \times L_{0}^{2}(\Omega)$ is a solution of problem $(1.1)-(2.2)$ if and only if it is a solution of problem (2.6).

We briefly recall from [17], $[5, \S 2],[6, \S 2.5]$ and $[4, \S 2]$ the main arguments for proving the existence of a solution of problem (2.6). It is readily checked that the kernel

$$
V=\left\{\boldsymbol{v} \in \mathbb{D}(\Omega) ; \forall q \in L_{0}^{2}(\Omega), b(\boldsymbol{v}, q)=0\right\},
$$


coincides with the space of divergence-free functions in $\mathbb{D}(\Omega)$. Similarly, the kernel

$$
\mathcal{W}=\left\{(\theta, \boldsymbol{w}) \in H_{0}^{1}(\Omega) \times V ; \forall \varphi \in H_{0}^{1}(\Omega), c(\theta, \boldsymbol{w} ; \varphi)=0\right\},
$$

coincides with the space of pairs $(\theta, \boldsymbol{w})$ in $H_{0}^{1}(\Omega) \times V$ such that $\theta$ is equal to curl $\boldsymbol{w}$ in the distribution sense. We observe that, for any solution $(\omega, \boldsymbol{u}, p)$ of problem (2.6), the pair $(\omega, \boldsymbol{u})$ is a solution of the following reduced problem

Find $(\omega, \boldsymbol{u})$ in $\mathcal{W}$ such that

$$
\forall \boldsymbol{v} \in V, \quad a(\omega, \boldsymbol{u} ; \boldsymbol{v})+K(\omega, \boldsymbol{u} ; \boldsymbol{v})=\langle\boldsymbol{f}, \boldsymbol{v}\rangle .
$$

We recall from [5, Lemma 2.3] and [6, Prop. 2.5.3 \& 2.5.4] the following properties (which require the further conditions on the $\Sigma_{j}$ as enforced in the definition of $\mathbb{D}(\Omega)$ ): There exists a positive constant $\alpha$ such that

$$
\begin{aligned}
\forall \boldsymbol{v} \in V \backslash\{0\}, & \sup _{(\omega, \boldsymbol{u}) \in \mathcal{W}} a(\omega, \boldsymbol{u} ; \boldsymbol{v})>0, \\
& \forall(\omega, \boldsymbol{u}) \in \mathcal{W}, \quad \sup _{\boldsymbol{v} \in V} \frac{a(\omega, \boldsymbol{u} ; \boldsymbol{v})}{\|\boldsymbol{v}\|_{L^{2}(\Omega)^{2}}} \geq \alpha\left(\|\omega\|_{H^{1}(\Omega)}^{2}+\|\boldsymbol{u}\|_{L^{2}(\Omega)^{2}}^{2}\right)^{\frac{1}{2}} .
\end{aligned}
$$

This last property is derived from the more precise inequality

$$
\forall(\omega, \boldsymbol{u}) \in \mathcal{W}, \quad a(\omega, \boldsymbol{u} ; \boldsymbol{u}+\operatorname{curl} \omega) \geq 2 \alpha\left(\|\omega\|_{H^{1}(\Omega)}^{2}+\|\boldsymbol{u}\|_{L^{2}(\Omega)^{2}}^{2}\right),
$$

which is used in the proof of the existence result below.

The next statement is an easy consequence of the imbedding of $\mathcal{W}$ into $H^{1}(\Omega) \times$ $H^{\frac{1}{2}}(\Omega)^{2}$, see [10], and of the Sobolev imbeddings of $H^{1}(\Omega)$ into $L^{q}(\Omega)$ for any $q<+\infty$ and of $H^{\frac{1}{2}}(\Omega)$ into $L^{4}(\Omega)$.

Lemma 2.3. The form $K(\cdot, \cdot ; \cdot)$ is continuous on $\mathcal{W} \times L^{2}(\Omega)^{2}$. Moreover, for any $(\omega, \boldsymbol{u})$ in $\mathcal{W}$, the operators: $(\theta, \boldsymbol{w}) \mapsto \omega \times \boldsymbol{w}$ and $(\theta, \boldsymbol{w}) \mapsto \theta \times \boldsymbol{u}$ are compact from $\mathcal{W}$ into $L^{2}(\Omega)^{2}$.

We note the further antisymmetry properties

$$
\forall(\omega, \boldsymbol{u}) \in \mathcal{W}, \quad K(\omega, \boldsymbol{u} ; \boldsymbol{u})=K(\omega, \boldsymbol{u} ; \operatorname{curl} \omega)=0,
$$

which allow to establish a priori estimates on any solution of problem (2.13). Thus, the existence of a solution for this problem is derived from Brouwer's fixed point theorem in a standard way, see [4, Prop. 2.5] for a detailed proof.

Proposition 2.4. For any data $\boldsymbol{f}$ in the dual space of $H_{0}(\operatorname{div}, \Omega)$, problem (2.13) has a solution $(\omega, \boldsymbol{u})$ in $\mathcal{W}$.

We also recall the standard inf-sup condition on the form $b(\cdot, \cdot)$ : There exists a positive constant $\beta$ such that

$$
\forall q \in L_{0}^{2}(\Omega), \quad \sup _{\boldsymbol{v} \in H_{0}(\operatorname{div}, \Omega)} \frac{b(\boldsymbol{v}, q)}{\|\boldsymbol{v}\|_{H(\operatorname{div}, \Omega)}} \geq \beta\|q\|_{L^{2}(\Omega)} .
$$


When applying this result with $\Omega$ replaced by $\Omega^{\circ}$, we easily derive that

$$
\forall q \in L_{0}^{2}(\Omega), \quad \sup _{\boldsymbol{v} \in \mathbb{D}(\Omega)} \frac{b(\boldsymbol{v}, q)}{\|\boldsymbol{v}\|_{H(\operatorname{div}, \Omega)}} \geq \beta\|q\|_{L^{2}(\Omega)} .
$$

Combining this with Proposition 2.4 leads to the main result for problem (2.6).

Theorem 2.5. For any data $\boldsymbol{f}$ in the dual space of $H_{0}(\operatorname{div}, \Omega)$, problem (2.6) has a solution $(\omega, \boldsymbol{u}, p)$ in $H_{0}^{1}(\Omega) \times \mathbb{D}(\Omega) \times L_{0}^{2}(\Omega)$. Moreover this solution satisfies

$$
\|\omega\|_{H^{1}(\Omega)}+\|\boldsymbol{u}\|_{H(\operatorname{div}, \Omega)}+\|p\|_{L^{2}(\Omega)} \leq c\|\boldsymbol{f}\|_{H_{0}(\operatorname{div}, \Omega)^{\prime}} .
$$

Remark 2.6. As usual for the Navier-Stokes equations, the solution of problem (2.6) is unique only if the viscosity $\nu$ is large enough as a function of the data, see [4, Thm 2.9]. We prefer to avoid this too restrictive assumption in what follows.

We conclude with some regularity properties of the solution of problem (2.6) which can easily be derived from $[2, \S 2]$ thanks to a boot-strap argument: The mapping: $\boldsymbol{f} \mapsto$ $(\omega, \boldsymbol{u}, p)$, where $(\omega, \boldsymbol{u}, p)$ is the solution of problem (2.6) with data $\boldsymbol{f}$, is continuous from $H^{s}(\Omega)^{2}$ into $H^{s+1}(\Omega) \times H^{s}(\Omega)^{2} \times H^{s+1}(\Omega)$, for

(i) all $s \leq \frac{1}{2}$ in the general case,

(ii) all $s \leq 1$ when $\Omega$ is convex,

(iii) all $s<\frac{\pi}{\alpha}$ when $\Omega$ is a polygon with largest angle equal to $\alpha$. 


\section{The spectral element discrete problem.}

From now on, we assume that $\Omega$ admits a partition without overlap into a finite number of subdomains

$$
\bar{\Omega}=\cup_{k=1}^{K} \Omega_{k} \quad \text { and } \quad \Omega_{k} \cap \Omega_{k^{\prime}}=\emptyset, \quad 1 \leq k<k^{\prime} \leq K,
$$

which satisfy the further conditions:

(i) Each $\Omega_{k}, 1 \leq k \leq K$, is a rectangle,

(ii) The intersection of two subdomains $\bar{\Omega}_{k}$ and $\bar{\Omega}_{k^{\prime}}, 1 \leq k<k^{\prime} \leq K$, if not empty, is either a vertex or a whole edge of both $\Omega_{k}$ and $\Omega_{k^{\prime}}$,

(iii) The $\bar{\Sigma}_{j}, 1 \leq j \leq J$, introduced in Notation 2.1, are the union of whole edges of some $\Omega_{k}$.

The discrete spaces are constructed from the finite elements proposed by Nédélec on cubic three-dimensional meshes, see $[15, \S 2]$. In order to describe them and for any pair $(m, n)$ of nonnegative integers, we introduce the space $\mathbb{P}_{m, n}\left(\Omega_{k}\right)$ of restrictions to $\Omega_{k}$ of polynomials with degree $\leq m$ with respect to $x$ and $\leq n$ with respect to $y$. When $m$ is equal to $n$, this space is simply denoted by $\mathbb{P}_{n}\left(\Omega_{k}\right)$. Relying on these definitions, we introduce the local spaces, for an integer $N \geq 2$,

$$
D_{N}^{k}=\mathbb{P}_{N, N-1}\left(\Omega_{k}\right) \times \mathbb{P}_{N-1, N}\left(\Omega_{k}\right), \quad C_{N}^{k}=\mathbb{P}_{N}\left(\Omega_{k}\right), \quad M_{N}^{k}=\mathbb{P}_{N-1}\left(\Omega_{k}\right) .
$$

The space $\mathbb{D}_{N}$ which approximates $\mathbb{D}(\Omega)$ is then defined by

$$
\mathbb{D}_{N}=\left\{\boldsymbol{v}_{N} \in \mathbb{D}(\Omega) ;\left.\boldsymbol{v}_{N}\right|_{\Omega_{k}} \in D_{N}^{k}, 1 \leq k \leq K\right\} .
$$

The space $\mathbb{C}_{N}$ which approximates $H_{0}^{1}(\Omega)$ is defined by

$$
\mathbb{C}_{N}=\left\{\varphi_{N} \in H_{0}^{1}(\Omega) ;\left.\varphi_{N}\right|_{\Omega_{k}} \in C_{N}^{k}, 1 \leq k \leq K\right\} .
$$

Finally, for the approximation of $L_{0}^{2}(\Omega)$, we consider the space

$$
\mathbb{M}_{N}=\left\{q_{N} \in L_{0}^{2}(\Omega) ;\left.q_{N}\right|_{\Omega_{k}} \in M_{N}^{k}, 1 \leq k \leq K\right\} .
$$

It can be noted that the functions in $\mathbb{D}_{N}$ have continuous normal traces through the interfaces $\bar{\Omega}_{k} \cap \bar{\Omega}_{k^{\prime}}$ while the functions in $\mathbb{C}_{N}$ have continuous traces. Thanks to the previous choice, the discretization that we propose is perfectly conforming.

According to the approach suggested in [14] and in order to handle the nonlinear term, we use over-integration. For a fixed real number $\mu, 0<\mu \leq 1$, we associate with each value of $N$ the quantity $m(N)$ equal to the integer part of $(1+\mu) N$. Setting $\xi_{0}=-1$ and $\xi_{m(N)}=1$, we introduce the $m(N)-1$ nodes $\xi_{j}, 1 \leq j \leq m(N)-1$, and the $m(N)+1$ weights $\rho_{j}, 0 \leq j \leq m(N)$, of the Gauss-Lobatto quadrature formula on $[-1,1]$. Denoting by $\mathbb{P}_{n}(-1,1)$ the space of restrictions to $[-1,1]$ of polynomials with degree $\leq n$, we recall that the following equality holds

$$
\forall \Phi \in \mathbb{P}_{2 m(N)-1}(-1,1), \quad \int_{-1}^{1} \Phi(\zeta) d \zeta=\sum_{j=0}^{m(N)} \Phi\left(\xi_{j}\right) \rho_{j} .
$$


We also recall $[7$, form. (13.20)] the following property, which is useful in what follows

$$
\forall \varphi_{N} \in \mathbb{P}_{m(N)}(-1,1), \quad\left\|\varphi_{N}\right\|_{L^{2}(-1,1)}^{2} \leq \sum_{j=0}^{m(N)} \varphi_{N}^{2}\left(\xi_{j}\right) \rho_{j} \leq 3\left\|\varphi_{N}\right\|_{L^{2}(-1,1)}^{2} .
$$

Denoting by $F_{k}$ the affine mapping that sends $]-1,1\left[{ }^{2}\right.$ onto $\Omega_{k}$, we introduce the local discrete products, defined on continuous functions $u$ and $v$ on $\bar{\Omega}_{k}$ by

$$
(u, v)_{N}^{k}=\frac{\operatorname{meas}\left(\Omega_{k}\right)}{4} \sum_{i=0}^{m(N)} \sum_{j=0}^{m(N)} u \circ F_{k}\left(\xi_{i}, \xi_{j}\right) v \circ F_{k}\left(\xi_{i}, \xi_{j}\right) \rho_{i} \rho_{j} .
$$

The global product is then defined on continuous functions $u$ and $v$ on $\bar{\Omega}$ by

$$
((u, v))_{N}=\sum_{k=1}^{K}\left(\left.u\right|_{\Omega_{k}},\left.v\right|_{\Omega_{k}}\right)_{N}^{k}
$$

We also need the local Lagrange interpolation operators $\mathcal{I}_{N}^{k}$ : For each function $\varphi$ continuous on $\bar{\Omega}_{k}, \mathcal{I}_{N}^{k} \varphi$ belongs to $\mathbb{P}_{m(N)}\left(\Omega_{k}\right)$ and is equal to $\varphi$ at all nodes $F_{k}\left(\xi_{i}, \xi_{j}\right), 0 \leq i, j \leq m(N)$. Finally, for each function $\varphi$ continuous on $\bar{\Omega}, \mathcal{I}_{N} \varphi$ denotes the function equal to $\left.\mathcal{I}_{N}^{k} \varphi\right|_{\Omega_{k}}$ on each $\Omega_{k}, 1 \leq k \leq K$.

The discrete problem is now constructed from (2.6) by using the Galerkin method combined with numerical integration. It reads

Find $\left(\omega_{N}, \boldsymbol{u}_{N}, p_{N}\right)$ in $\mathbb{C}_{\mathbb{N}} \times \mathbb{D}_{N} \times \mathbb{M}_{N}$ such that

$$
\begin{array}{ll}
\forall \boldsymbol{v}_{N} \in \mathbb{D}_{N}, \quad a_{N}\left(\omega_{N}, \boldsymbol{u}_{N} ; \boldsymbol{v}_{N}\right)+K_{N}\left(\omega_{N}, \boldsymbol{u}_{N} ; \boldsymbol{v}_{N}\right)+b_{N}\left(\boldsymbol{v}_{N}, p_{N}\right)=\left(\left(\boldsymbol{f}, \boldsymbol{v}_{N}\right)\right)_{N}, \\
\forall q_{N} \in \mathbb{M}_{N}, \quad b_{N}\left(\boldsymbol{u}_{N}, q_{N}\right)=0, \\
\forall \varphi_{N} \in \mathbb{C}_{N}, \quad c_{N}\left(\omega_{N}, \boldsymbol{u}_{N} ; \varphi_{N}\right)=0,
\end{array}
$$

where the bilinear forms $a_{N}(\cdot, \cdot ; \cdot), b_{N}(\cdot, \cdot)$ and $c_{N}(\cdot, \cdot ; \cdot)$ are defined by

$$
\begin{array}{r}
a_{N}\left(\omega_{N}, \boldsymbol{u}_{N} ; \boldsymbol{v}_{N}\right)=\nu\left(\left(\operatorname{curl} \omega_{N}, \boldsymbol{v}_{N}\right)\right)_{N}, \quad b_{N}\left(\boldsymbol{v}_{N}, q_{N}\right)=-\left(\left(\operatorname{div} \boldsymbol{v}_{N}, q_{N}\right)\right)_{N}, \\
c_{N}\left(\omega_{N}, \boldsymbol{u}_{N} ; \varphi_{N}\right)=\left(\left(\omega_{N}, \varphi_{N}\right)\right)_{N}-\left(\left(\boldsymbol{u}_{N}, \operatorname{curl} \varphi_{N}\right)\right)_{N},
\end{array}
$$

while the trilinear form $K_{N}(\cdot, \cdot ; \cdot \cdot)$ is now given by

$$
K_{N}\left(\omega_{N}, \boldsymbol{u}_{N} ; \boldsymbol{v}_{N}\right)=\left(\left(\omega_{N} \times \boldsymbol{u}_{N}, \boldsymbol{v}_{N}\right)\right)_{N} .
$$

As a consequence of the exactness property $(3.6)$, the forms $a(\cdot, \cdot ; \cdot)$ and $a_{N}(\cdot, \cdot ; \cdot)$, and also $c(\cdot, \cdot ; \cdot)$ and $c_{N}(\cdot, \cdot ; \cdot)$ coincide on $\left(\mathbb{C}_{N} \times \mathbb{D}_{N}\right) \times \mathbb{D}_{N}$ and $\left(\mathbb{C}_{N} \times \mathbb{D}_{N}\right) \times \mathbb{C}_{N}$, respectively, when $m(N)>N$. Moreover, the forms $b(\cdot, \cdot)$ and $b_{N}(\cdot, \cdot)$ coincide on $\mathbb{D}_{N} \times \mathbb{M}_{N}$ even for $m(N)=N$. In any case, it follows from (3.7) combined with Cauchy-Schwarz inequalities that the forms $a_{N}(\cdot, \cdot ; \cdot), b_{N}(\cdot, \cdot)$ and $c_{N}(\cdot, \cdot ; \cdot)$ are continuous on $\left(\mathbb{C}_{N} \times \mathbb{D}_{N}\right) \times \mathbb{D}_{N}, \mathbb{D}_{N} \times \mathbb{M}_{N}$ and $\left(\mathbb{C}_{N} \times \mathbb{D}_{N}\right) \times \mathbb{C}_{N}$, respectively, with norms bounded independently of $N$. 
In order to perform the numerical analysis of problem (3.10), we first recall from the finite element analogous result [15] that the range of $\mathbb{D}_{N}$ by the divergence operator is contained in $\mathbb{M}_{N}$. So, if $V_{N}$ denotes the kernel

$$
V_{N}=\left\{\boldsymbol{v}_{N} \in \mathbb{D}_{N} ; \forall q_{N} \in \mathbb{M}_{N}, b_{N}\left(\boldsymbol{v}_{N}, q_{N}\right)=0\right\}
$$

it is readily checked by taking $q_{N}$ equal to $\operatorname{div} \boldsymbol{v}_{N}$ in the previous line that $V_{N}$ is the space of divergence-free functions in $\mathbb{D}_{N}$, i.e. coincides with $\mathbb{D}_{N} \cap V$. Similarly, we introduce the discrete kernel

$$
\mathcal{W}_{N}=\left\{\left(\theta_{N}, \boldsymbol{w}_{N}\right) \in \mathbb{C}_{N} \times V_{N} ; \forall \varphi_{N} \in \mathbb{C}_{N}, c_{N}\left(\theta_{N}, \boldsymbol{w}_{N} ; \varphi_{N}\right)=0\right\}
$$

We observe that, for any solution $\left(\omega_{N}, \boldsymbol{u}_{N}, p_{N}\right)$ of problem $(3.10)$, the pair $\left(\omega_{N}, \boldsymbol{u}_{N}\right)$ is a solution of the reduced problem

Find $\left(\omega_{N}, \boldsymbol{u}_{N}\right)$ in $\mathcal{W}_{N}$, such that

$$
\forall \boldsymbol{v}_{N} \in V_{N}, \quad a_{N}\left(\omega_{N}, \boldsymbol{u}_{N} ; \boldsymbol{v}_{N}\right)+K_{N}\left(\omega_{N}, \boldsymbol{u}_{N} ; \boldsymbol{v}_{N}\right)=\left(\left(\boldsymbol{f}, \boldsymbol{v}_{N}\right)\right)_{N}
$$

We first recall the next result which is proved in [2, Prop. 3.3].

Lemma 3.1. For each $\boldsymbol{v}_{N}$ in $V_{N}$, there exists a unique $\psi_{N}$ in $\mathbb{C}_{N}$ such that $\boldsymbol{v}_{N}=\operatorname{curl} \psi_{N}$ and which satisfies

$$
\left\|\psi_{N}\right\|_{L^{2}(\Omega)} \leq c\left\|\boldsymbol{v}_{N}\right\|_{L^{2}(\Omega)^{2}}
$$

Relying on (3.16), we prove the following property.

Lemma 3.2. There exists a constant $\alpha_{*}$ such that

$$
\forall\left(\omega_{N}, \boldsymbol{u}_{N}\right) \in \mathcal{W}_{N}, \quad a_{N}\left(\omega_{N}, \boldsymbol{u}_{N} ; \boldsymbol{u}_{N}\right) \geq \alpha_{*}\left(\left\|\omega_{N}\right\|_{L^{2}(\Omega)}^{2}+\left\|\boldsymbol{u}_{N}\right\|_{L^{2}(\Omega)^{2}}^{2}\right)
$$

Proof: From the definitions of the form $a_{N}(\cdot, \cdot ; \cdot)$ and the space $\mathcal{W}_{N}$, we have

$$
a_{N}\left(\omega_{N}, \boldsymbol{u}_{N} ; \boldsymbol{u}_{N}\right)=\nu\left(\left(\operatorname{curl} \omega_{N}, \boldsymbol{u}_{N}\right)\right)_{N}=\nu\left(\left(\omega_{N}, \omega_{N}\right)\right)_{N},
$$

whence, owing to $(3.7)$,

$$
a_{N}\left(\omega_{N}, \boldsymbol{u}_{N} ; \boldsymbol{u}_{N}\right) \geq \nu\left\|\omega_{N}\right\|_{L^{2}(\Omega)}^{2} .
$$

On the other hand, associating with $\boldsymbol{u}_{N}$ the function $\psi_{N}$ exhibited in Lemma 3.1 and using once more the definition of $\mathcal{W}_{N}$, we have

$$
\left(\left(\boldsymbol{u}_{N}, \boldsymbol{u}_{N}\right)\right)_{N}=\left(\left(\operatorname{curl} \psi_{N}, \boldsymbol{u}_{N}\right)\right)_{N}=\left(\left(\omega_{N}, \psi_{N}\right)\right)_{N}
$$

Combining (3.7) with (3.16) yields

$$
\left\|\boldsymbol{u}_{N}\right\|_{L^{2}(\Omega)^{2}}^{2} \leq 9\left\|\omega_{N}\right\|_{L^{2}(\Omega)}\left\|\psi_{N}\right\|_{L^{2}(\Omega)} \leq 9 c\left\|\omega_{N}\right\|_{L^{2}(\Omega)}\left\|\boldsymbol{u}_{N}\right\|_{L^{2}(\Omega)^{2}}
$$


whence

$$
\left\|\boldsymbol{u}_{N}\right\|_{L^{2}(\Omega)^{2}} \leq 9 c\left\|\omega_{N}\right\|_{L^{2}(\Omega)} .
$$

This last inequality and (3.18) give the desired property.

We are now in a position to prove the existence of a solution to problem (3.15).

Proposition 3.3. For any function $\boldsymbol{f}$ continuous on $\bar{\Omega}$, problem (3.15) has a solution $\left(\omega_{N}, \boldsymbol{u}_{N}\right)$ in $\mathcal{W}_{N}$. Moreover this solution satisfies for a constant $c$ independent of $N$

$$
\left\|\omega_{N}\right\|_{L^{2}(\Omega)}+\left\|\boldsymbol{u}_{N}\right\|_{L^{2}(\Omega)^{2}} \leq c\left\|\mathcal{I}_{N} \boldsymbol{f}\right\|_{L^{2}(\Omega)^{2}} .
$$

Proof: We introduce the mapping $\Phi_{N}$ defined from $\mathcal{W}_{N}$ into its dual space by

$$
\begin{aligned}
& \forall\left(\omega_{N}, \boldsymbol{u}_{N}\right) \in \mathcal{W}_{N}, \forall\left(\boldsymbol{\vartheta}_{N}, \boldsymbol{w}_{N}\right) \in \mathcal{W}_{N}, \\
& \left\langle\Phi_{N}\left(\omega_{N}, \boldsymbol{u}_{N}\right),\left(\boldsymbol{\vartheta}_{N}, \boldsymbol{w}_{N}\right)\right\rangle=a_{N}\left(\omega_{N}, \boldsymbol{u}_{N} ; \boldsymbol{w}_{N}\right)+K_{N}\left(\omega_{N}, \boldsymbol{u}_{N} ; \boldsymbol{w}_{N}\right)-\left(\boldsymbol{f}, \boldsymbol{w}_{N}\right)_{N} .
\end{aligned}
$$

The space $\mathcal{W}_{N}$ is for a while provided with the weak norm

$$
\left(\left\|\omega_{N}\right\|_{L^{2}(\Omega)}^{2}+\left\|\boldsymbol{u}_{N}\right\|_{L^{2}(\Omega)^{2}}^{2}\right)^{\frac{1}{2}}
$$

Since $\mathcal{W}_{N}$ is finite-dimensional, it is readily checked that $\Phi_{N}$ is continuous. Next, noting that $K_{N}\left(\omega_{N}, \boldsymbol{u}_{N} ; \boldsymbol{u}_{N}\right)$ is zero (indeed, the product $\left(\omega_{N} \times \boldsymbol{u}_{N}\right) \cdot \boldsymbol{u}_{N}$ vanishes at all nodes $\left.F_{k}\left(\xi_{i}, \xi_{j}\right)\right)$, we have

$$
\left\langle\Phi_{N}\left(\omega_{N}, \boldsymbol{u}_{N}\right),\left(\omega_{N}, \boldsymbol{u}_{N}\right)\right\rangle=a_{N}\left(\omega_{N}, \boldsymbol{u}_{N} ; \boldsymbol{u}_{N}\right)-\left(\boldsymbol{f}, \boldsymbol{u}_{N}\right)_{N},
$$

whence, owing to Lemma 3.2,

$$
\left\langle\Phi_{N}\left(\omega_{N}, \boldsymbol{u}_{N}\right),\left(\omega_{N}, \boldsymbol{u}_{N}\right)\right\rangle \geq \alpha_{*}\left(\left\|\omega_{N}\right\|_{L^{2}(\Omega)}^{2}+\left\|\boldsymbol{u}_{N}\right\|_{L^{2}(\Omega)^{2}}^{2}\right)-\left(\boldsymbol{f}, \boldsymbol{u}_{N}\right)_{N} .
$$

On the other hand, we derive from (3.7) that

$$
\left(\boldsymbol{f}, \boldsymbol{u}_{N}\right)_{N}=\left(\mathcal{I}_{N} \boldsymbol{f}, \boldsymbol{u}_{N}\right)_{N} \leq 3\left\|\mathcal{I}_{N} \boldsymbol{f}\right\|_{L^{2}(\Omega)^{2}}\left\|\boldsymbol{u}_{N}\right\|_{L^{2}(\Omega)^{2}},
$$

which leads to

$$
\left\langle\Phi_{N}\left(\omega_{N}, \boldsymbol{u}_{N}\right),\left(\omega_{N}, \boldsymbol{u}_{N}\right)\right\rangle \geq \frac{\alpha_{*}}{2}\left(\left\|\omega_{N}\right\|_{L^{2}(\Omega)}^{2}+\left\|\boldsymbol{u}_{N}\right\|_{L^{2}(\Omega)^{2}}^{2}\right)-\frac{9}{2 \alpha^{*}}\left\|\mathcal{I}_{N} \boldsymbol{f}\right\|_{L^{2}(\Omega)^{2}}^{2} .
$$

Thus, setting

$$
\mu_{N}=\frac{3}{\alpha_{*}}\left\|\mathcal{I}_{N} \boldsymbol{f}\right\|_{L^{2}(\Omega)^{2}}
$$

we observe that $\left\langle\Phi_{N}\left(\omega_{N}, \boldsymbol{u}_{N}\right),\left(\omega_{N}, \boldsymbol{u}_{N}\right)\right\rangle$ is nonnegative on the sphere of $\mathcal{W}_{N}$ with radius $\mu_{N}$. So applying Brouwer's fixed point theorem, see [13, Chap. IV, Cor. 1.1], gives the existence result together with estimate (3.19). 
To go further, we recall from [2, Lemma 3.8] the inf-sup condition on the form $b_{N}(\cdot, \cdot)$ : There exists a positive constant $\beta_{*}$ independent of $N$ such that the form $b_{N}(\cdot, \cdot ; \cdot)$ satisfies the inf-sup condition

$$
\forall q_{N} \in \mathbb{M}_{N}, \quad \sup _{\boldsymbol{v}_{N} \in \mathbb{D}_{N}} \frac{b_{N}\left(\boldsymbol{v}_{N}, q_{N}\right)}{\left\|\boldsymbol{v}_{N}\right\|_{H(\operatorname{div}, \Omega)}} \geq \beta_{*}\left\|q_{N}\right\|_{L^{2}(\Omega)} .
$$

The final existence result is derived from this condition and Proposition 3.3 in a standard way, see [13, Chap. I, Lemma 4.1] for instance.

Theorem 3.4. For any function $\boldsymbol{f}$ continuous on $\bar{\Omega}$, problem (3.10) has a solution $\left(\omega_{N}, \boldsymbol{u}_{N}, p_{N}\right)$ in $\mathbb{C}_{N} \times \mathbb{D}_{N} \times \mathbb{M}_{N}$. Moreover the part $\left(\omega_{N}, \boldsymbol{u}_{N}\right)$ of this solution satisfies (3.19).

Note that all the results in this section hold without over-integration, i.e., for $\mu=0$ and $m(N)=N$. However, the choice of a $\mu>0$ is needed in what follows. 


\section{Error estimates.}

As already hinted, the error analysis of the discrete problem relies on the Brezzi, Rappaz and Raviart theory [8]. In order to apply it, we first write both problems (2.13) and (3.15) in a different form.

We set

$$
\mathcal{X}=H_{0}^{1}(\Omega) \times V
$$

Owing to the characterization of $V$, this space is equipped with the norm

$$
\|(\theta, \boldsymbol{w})\|_{\mathcal{X}}=\left(|\theta|_{H^{1}(\Omega)}^{2}+\|\boldsymbol{w}\|_{L^{2}(\Omega)^{2}}^{2}\right)^{\frac{1}{2}}
$$

Let $\mathcal{S}$ denote the following Stokes operator: For any data $\boldsymbol{f}$ in the dual space of $H_{0}(\operatorname{div}, \Omega)$, $\mathcal{S} \boldsymbol{f}$ denotes the solution $(\omega, \boldsymbol{u})$ of the reduced problem

Find $(\omega, \boldsymbol{u})$ in $\mathcal{W}$ such that

$$
\forall \boldsymbol{v} \in V, \quad a(\omega, \boldsymbol{u} ; \boldsymbol{v})=\langle\boldsymbol{f}, \boldsymbol{v}\rangle
$$

The fact that $\mathcal{S}$ is well-defined is easily derived from properties (2.14). We also introduce the mapping $G$ defined from $\mathcal{W}$ into the dual space of $H_{0}(\operatorname{div}, \Omega)$ by

$$
\forall(\omega, \boldsymbol{u}) \in \mathcal{W}, \forall \boldsymbol{v} \in H_{0}(\operatorname{div}, \Omega), \quad\langle G(\omega, \boldsymbol{u}), \boldsymbol{v}\rangle=K(\omega, \boldsymbol{u} ; \boldsymbol{v})-\langle\boldsymbol{f}, \boldsymbol{v}\rangle .
$$

Then, problem (2.13) can equivalently be written as

$$
(\omega, \boldsymbol{u})+\mathcal{S} G(\omega, \boldsymbol{u})=0
$$

Similarly, we set

$$
\mathcal{X}_{N}=\mathbb{C}_{N} \times V_{N}
$$

and note, since $V_{N}$ is contained in $V, \mathcal{X}_{N}$ is a finite-dimensional subspace of $\mathcal{X}$. It is still provided with the norm defined in (4.1). We thus define the discrete Stokes operator: For any data $\boldsymbol{f}$ in the dual space of $H_{0}(\operatorname{div}, \Omega), \mathcal{S}_{N} \boldsymbol{f}$ denotes the solution $\left(\omega_{N}, \boldsymbol{u}_{N}\right)$ of the problem

Find $\left(\omega_{N}, \boldsymbol{u}_{N}\right)$ in $\mathcal{W}_{N}$ such that

$$
\forall \boldsymbol{v}_{N} \in V_{N}, \quad a_{N}\left(\omega_{N}, \boldsymbol{u}_{N} ; \boldsymbol{v}_{N}\right)=\left\langle\boldsymbol{f}, \boldsymbol{v}_{N}\right\rangle
$$

The well-posedness of such a problem is proved in [2, Cor. 3.6] for a slightly different right-hand side. Finally we consider the mapping $G_{N}$ defined from $\mathcal{X}_{N}$ into the dual space of $\mathbb{D}_{N}$ by

$$
\begin{aligned}
\forall\left(\omega_{N}, \boldsymbol{u}_{N}\right) \in \mathcal{X}_{N}, & \forall \boldsymbol{v}_{N} \in \mathbb{D}_{N} \\
& \left\langle G_{N}\left(\omega_{N}, \boldsymbol{u}_{N}\right), \boldsymbol{v}_{N}\right\rangle=K_{N}\left(\omega_{N}, \boldsymbol{u}_{N} ; \boldsymbol{v}_{N}\right)-\left(\left(\boldsymbol{f}, \boldsymbol{v}_{N}\right)\right)_{N} .
\end{aligned}
$$


Then, problem (3.15) can equivalently be written as

$$
\left(\omega_{N}, \boldsymbol{u}_{N}\right)+\mathcal{S}_{N} G_{N}\left(\omega_{N}, \boldsymbol{u}_{N}\right)=0 .
$$

Using analogous arguments as in [2, Cor. $3.6 \& 4.9]$, we easily derive the following results:

(i) The operator $\mathcal{S}_{N}$ satisfies the stability property

$$
\left\|\mathcal{S}_{N} \boldsymbol{f}\right\|_{\mathcal{X}} \leq c \sup _{\boldsymbol{v}_{N} \in V_{N}} \frac{\left\langle\boldsymbol{f}, \boldsymbol{v}_{N}\right\rangle}{\left\|\boldsymbol{v}_{N}\right\|_{L^{2}(\Omega)^{2}}} ;
$$

(ii) The next error estimate for all $\boldsymbol{f}$ such that $\mathcal{S} \boldsymbol{f}$ belongs to $H^{s+1}(\Omega) \times H^{s}(\Omega)^{2}, s \geq 0$,

$$
\left\|\left(\mathcal{S}-\mathcal{S}_{N}\right) \boldsymbol{f}\right\|_{\mathcal{X}} \leq c N^{-s}\|\mathcal{S} \boldsymbol{f}\|_{H^{s+1}(\Omega) \times H^{s}(\Omega)^{2}} .
$$

We need some further properties of the form $K(\cdot, \cdot ; \cdot)$. The following result is derived in $[4$, Lemma 3.4] in the case of one subdomain $\Omega_{k}$ and relies on the following inverse inequality

$$
\forall \varphi_{N} \in \mathbb{P}_{N}\left(\Omega_{k}\right), \quad\left\|\varphi_{N}\right\|_{L^{\infty}\left(\Omega_{k}\right)} \leq c|\log N|^{\frac{1}{2}}\left\|\varphi_{N}\right\|_{H^{1}\left(\Omega_{k}\right)} .
$$

Applying the same arguments on each $\Omega_{k}$ leads to the next statement.

Lemma 4.1. The following property holds

$$
\begin{aligned}
\forall \omega_{N} \in \mathbb{C}_{N}, \forall \boldsymbol{u}_{N} \in \mathbb{D}_{N}, \forall \boldsymbol{v}_{N} & \in \mathbb{D}_{N}, \\
\left|K\left(\omega_{N}, \boldsymbol{u}_{N} ; \boldsymbol{v}_{N}\right)\right| & \leq c|\log N|^{\frac{1}{2}}\left\|\omega_{N}\right\|_{H^{1}(\Omega)}\left\|\boldsymbol{u}_{N}\right\|_{L^{2}(\Omega)^{2}}\left\|\boldsymbol{v}_{N}\right\|_{L^{2}(\Omega)^{2}} .
\end{aligned}
$$

Remark 4.2. Similar arguments yield that estimate (4.11) still holds when at most two of the three functions $\omega_{N}, \boldsymbol{u}_{N}$ and $\boldsymbol{v}_{N}$ are replaced by their analogues $\omega$ in $H_{0}^{1}(\Omega), \boldsymbol{u}$ and $\boldsymbol{v}$ in $\mathbb{D}(\Omega)$.

We need the analogous result for the form $K_{N}(\cdot, \cdot ; \cdot)$.

Lemma 4.3. The following property holds

$$
\begin{aligned}
\forall \omega_{N} & \in \mathbb{C}_{N}, \forall \boldsymbol{u}_{N} \in \mathbb{D}_{N}, \forall \boldsymbol{v}_{N} \in \mathbb{D}_{N}, \\
& \left|K_{N}\left(\omega_{N}, \boldsymbol{u}_{N} ; \boldsymbol{v}_{N}\right)\right| \leq c|\log N|^{\frac{1}{2}}\left\|\omega_{N}\right\|_{H^{1}(\Omega)}\left\|\boldsymbol{u}_{N}\right\|_{L^{2}(\Omega)^{2}}\left\|\boldsymbol{v}_{N}\right\|_{L^{2}(\Omega)^{2}} .
\end{aligned}
$$

Proof: We have, with obvious notation,

$$
\begin{aligned}
& K_{N}\left(\omega_{N}, \boldsymbol{u}_{N} ; \boldsymbol{v}_{N}\right)=\left(\left(\omega_{N} u_{N y}, v_{N x}\right)\right)_{N}-\left(\left(\omega_{N} u_{N x}, v_{N y}\right)\right)_{N} \\
& \quad=\left(\left(\mathcal{I}_{N}\left(\omega_{N} u_{N y}\right), v_{N x}\right)\right)_{N}-\left(\left(\mathcal{I}_{N}\left(\omega_{N} u_{N x}\right), v_{N y}\right)\right)_{N} .
\end{aligned}
$$

By combining Cauchy-Schwarz inequalities with (3.7), we obtain

$$
\left|K_{N}\left(\omega_{N}, \boldsymbol{u}_{N} ; \boldsymbol{v}_{N}\right)\right| \leq c\left\|\mathcal{I}_{N}\left(\omega_{N} \boldsymbol{u}_{N}\right)\right\|_{L^{2}(\Omega)^{2}}\left\|\boldsymbol{v}_{N}\right\|_{L^{2}(\Omega)^{2}} .
$$


The following result can easily derived from its one-dimensional analogue, see [7, form. (13.28)],

$$
\forall \varphi_{M} \in \mathbb{P}_{M}\left(\Omega_{k}\right), \quad\left\|\mathcal{I}_{N}^{k} \varphi_{M}\right\|_{L^{2}\left(\Omega_{k}\right)} \leq c\left(1+\frac{M}{m(N)}\right)^{2}\left\|\varphi_{M}\right\|_{L^{2}\left(\Omega_{k}\right)}
$$

Since both products $\left(\omega_{N} u_{N x}\right)_{\mid \Omega_{k}}$ and $\left(\omega_{N} u_{N y}\right)_{\mid \Omega_{k}}$ belong to $\mathbb{P}_{2 N}\left(\Omega_{k}\right)$ and the ratio $\frac{2 N}{m(N)}$ is smaller than 2 , this gives

$$
\left|K_{N}\left(\omega_{N}, \boldsymbol{u}_{N} ; \boldsymbol{v}_{N}\right)\right| \leq c\left\|\omega_{N} \boldsymbol{u}_{N}\right\|_{L^{2}(\Omega)^{2}}\left\|\boldsymbol{v}_{N}\right\|_{L^{2}(\Omega)^{2}}
$$

We conclude by using the inequality

$$
\left\|\omega_{N} \boldsymbol{u}_{N}\right\|_{L^{2}(\Omega)^{2}} \leq\left\|\omega_{N}\right\|_{L^{\infty}(\Omega)}\left\|\boldsymbol{u}_{N}\right\|_{L^{2}(\Omega)^{2}}
$$

together with (4.10).

We are led to make the following assumptions. Here, $D$ stands for the differential operator.

Assumption 4.4. The triple $(\omega, \boldsymbol{u}, p)$ is a solution of problem (2.6) such that the operator Id $+\mathcal{S} D G(\omega, \boldsymbol{u})$ is an isomorphism of $\mathcal{X}$.

Note that this assumption can equivalently be written as follows (this requires the inf-sup condition (2.16)): For any data $\boldsymbol{g}$ in the dual space of $H_{0}(\operatorname{div}, \Omega)$, the linearized problem

Find $(\theta, \boldsymbol{w}, r)$ in $H_{0}^{1}(\Omega) \times H_{0}(\operatorname{div}, \Omega) \times L_{0}^{2}(\Omega)$ such that

$$
\begin{aligned}
& \forall \boldsymbol{v} \in H_{0}(\operatorname{div}, \Omega), \quad a(\theta, \boldsymbol{w} ; \boldsymbol{v})+K(\omega, \boldsymbol{w} ; \boldsymbol{v})+K(\theta, \boldsymbol{u} ; \boldsymbol{v})+b(\boldsymbol{v}, r)=\langle\boldsymbol{g}, \boldsymbol{v}\rangle, \\
& \forall q \in L_{0}^{2}(\Omega), \quad b(\boldsymbol{w}, q)=0, \\
& \forall \varphi \in H_{0}^{1}(\Omega), \quad c(\theta, \boldsymbol{w} ; \varphi)=0,
\end{aligned}
$$

has a unique solution with norm bounded by a constant times $\|\boldsymbol{g}\|_{H_{0}(\operatorname{div}, \Omega)^{\prime}}$. It yields the local uniqueness of the solution $(\omega, \boldsymbol{u}, p)$ but is much less restrictive than the conditions for its global uniqueness, see Remark 2.6.

Assumption 4.5. The solution $(\omega, \boldsymbol{u}, p)$ of problem (2.6) introduced in Assumption 4.4 belongs to $H^{s+1}(\Omega) \times H^{s}(\Omega)^{2} \times H^{s}(\Omega), s>0$.

Relying on this last assumption and taking $\tilde{N}$ equal to the integer part of $2 \mu N-1$, we can also construct from the arguments in $[2, \S 4]$ a pair $\left(\tilde{\omega}_{N}, \tilde{\boldsymbol{u}}_{N}\right)$ in $\mathbb{C}_{\tilde{N}} \times V_{\tilde{N}}$ (with obvious definitions for these new spaces) which satisfies

$$
\left\|\left(\omega-\tilde{\omega}_{N}, \boldsymbol{u}-\tilde{\boldsymbol{u}}_{N}\right)\right\|_{\mathcal{X}} \leq c \tilde{N}^{-s}\|(\omega, \boldsymbol{u})\|_{H^{s+1}(\Omega) \times H^{s}(\Omega)^{2}} .
$$

Note that estimate (4.14) makes sense only when $\tilde{N} \geq 2$. 
Let $\mathcal{L}\left(\mathcal{X}_{N}\right)$ denote the space of endomorphisms on $\mathcal{X}_{N}$. We are now in a position to state and prove the following lemma.

Lemma 4.6. If Assumptions 4.4 and 4.5 are satisfied, there exists an integer $N_{0}$ such that, for all $N \geq N_{0}$, the operator Id $+\mathcal{S}_{N} D G_{N}\left(\tilde{\omega}_{N}, \tilde{\boldsymbol{u}}_{N}\right)$ is an isomorphism of $\mathcal{X}_{N}$. Moreover the norm of its inverse operator is bounded independently of $N$.

Proof: We write the expansion

$$
\begin{aligned}
& \operatorname{Id}+\mathcal{S}_{N} D G_{N}\left(\tilde{\omega}_{N}, \tilde{\boldsymbol{u}}_{N}\right)=\operatorname{Id}+\mathcal{S} D G(\omega, \boldsymbol{u})-\left(\mathcal{S}-\mathcal{S}_{N}\right) D G(\omega, \boldsymbol{u}) \\
& \quad-\mathcal{S}_{N}\left(D G(\omega, \boldsymbol{u})-D G\left(\tilde{\omega}_{N}, \tilde{\boldsymbol{u}}_{N}\right)\right)-\mathcal{S}_{N}\left(D G\left(\tilde{\omega}_{N}, \tilde{\boldsymbol{u}}_{N}\right)-D G_{N}\left(\tilde{\omega}_{N}, \tilde{\boldsymbol{u}}_{N}\right)\right) .
\end{aligned}
$$

Moreover, it follows from the definition of $G$ and $G_{N}$ that, for all $\left(\theta_{N}, \boldsymbol{w}_{N}\right)$ in $\mathcal{X}_{N}$ and $\boldsymbol{v}_{N}$ in $V_{N}$,

$$
\begin{aligned}
& \left\langle D G\left(\tilde{\omega}_{N}, \tilde{\boldsymbol{u}}_{N}\right) \cdot\left(\theta_{N}, \boldsymbol{w}_{N}\right), \boldsymbol{v}_{N}\right\rangle=K\left(\tilde{\omega}_{N}, \boldsymbol{w}_{N} ; \boldsymbol{v}_{N}\right)+K\left(\theta_{N}, \tilde{\boldsymbol{u}}_{N} ; \boldsymbol{v}_{N}\right), \\
& \left\langle D G_{N}\left(\tilde{\omega}_{N}, \tilde{\boldsymbol{u}}_{N}\right) \cdot\left(\theta_{N}, \boldsymbol{w}_{N}\right), \boldsymbol{v}_{N}\right\rangle=K_{N}\left(\tilde{\omega}_{N}, \boldsymbol{w}_{N} ; \boldsymbol{v}_{N}\right)+K_{N}\left(\theta_{N}, \tilde{\boldsymbol{u}}_{N} ; \boldsymbol{v}_{N}\right) .
\end{aligned}
$$

Owing to the choice of $\left(\tilde{\omega}_{N}, \tilde{\boldsymbol{u}}_{N}\right)$, all products $\left.\left(\left(\tilde{\omega}_{N} \times \boldsymbol{w}_{N}\right) \cdot \boldsymbol{v}_{N}\right)\right|_{\Omega_{k}}$ and $\left.\left(\left(\theta_{N} \times \tilde{\boldsymbol{u}}_{N}\right) \cdot \boldsymbol{v}_{N}\right)\right|_{\Omega_{k}}$ belong to $\mathbb{P}_{2 m(N)-1}\left(\Omega_{k}\right)$, so that the last term in (4.15) vanishes. By combining (4.8) and Lemma 4.1, we also have

$$
\begin{aligned}
& \left\|\mathcal{S}_{N}\left(D G(\omega, \boldsymbol{u})-D G\left(\tilde{\omega}_{N}, \tilde{\boldsymbol{u}}_{N}\right)\right) \cdot\left(\theta_{N}, \boldsymbol{w}_{N}\right)\right\|_{\mathcal{X}} \\
& \quad \leq c|\log N|^{\frac{1}{2}}\left(\left\|\omega-\tilde{\omega}_{N}\right\|_{H^{1}(\Omega)}\left\|\boldsymbol{w}_{N}\right\|_{L^{2}(\Omega)^{2}}+\left\|\theta_{N}\right\|_{H^{1}(\Omega)}\left\|\boldsymbol{u}-\tilde{\boldsymbol{u}}_{N}\right\|_{L^{2}(\Omega)^{2}}\right) .
\end{aligned}
$$

Thus, applying estimate (4.14) yields

$$
\lim _{N \rightarrow+\infty}\left\|\mathcal{S}_{N}\left(D G(\omega, \boldsymbol{u})-D G\left(\tilde{\omega}_{N}, \tilde{\boldsymbol{u}}_{N}\right)\right)\right\|_{\mathcal{L}\left(\mathcal{X}_{N}\right)}=0
$$

Finally, it follows from Assumption 4.5 that, when $(\theta, \boldsymbol{w})$ runds through the unit ball of $\mathcal{X}, D G(\omega, \boldsymbol{u}) .(\theta, \boldsymbol{w})$ belongs to a compact of $L^{2}(\Omega)^{2}$, so that the next property is derived from (4.8) and (4.9) by standard arguments

$$
\lim _{N \rightarrow+\infty}\left\|\left(\mathcal{S}-\mathcal{S}_{N}\right) D G(\omega, \boldsymbol{u})\right\|_{\mathcal{L}\left(\mathcal{X}_{N}\right)}=0
$$

Thanks to Assumption 4.4, if $\gamma$ denotes the norm of the inverse of $\operatorname{Id}+\mathcal{S} D G(\omega, \boldsymbol{u})$, choosing $N$ large enough for the quantities in (4.16) and (4.17) to be smaller than $\frac{1}{4 \gamma}$ gives the desired property with the norm of the inverse of $\operatorname{Id}+\mathcal{S}_{N} D G_{N}\left(\tilde{\omega}_{N}, \tilde{\boldsymbol{u}}_{N}\right)$ smaller than $2 \gamma$.

Lemma 4.7. The following Lipschitz property holds

$$
\begin{aligned}
& \forall\left(\omega_{N}^{*}, \boldsymbol{u}_{N}^{*}\right) \in \mathcal{X}_{N}, \\
& \quad\left\|\mathcal{S}_{N}\left(D G_{N}\left(\tilde{\omega}_{N}, \tilde{\boldsymbol{u}}_{N}\right)-D G_{N}\left(\omega_{N}^{*}, \boldsymbol{u}_{N}^{*}\right)\right)\right\|_{\mathcal{L}\left(\mathcal{X}_{N}\right)} \leq c|\log N|^{\frac{1}{2}}\left\|\left(\tilde{\omega}_{N}-\omega_{N}^{*}, \tilde{\boldsymbol{u}}_{N}-\boldsymbol{u}_{N}^{*}\right)\right\|_{\mathcal{X}} .
\end{aligned}
$$


Proof: We have

$$
\begin{aligned}
\left\langle\left(D G_{N}\left(\tilde{\omega}_{N}, \tilde{\boldsymbol{u}}_{N}\right)-D G_{N}\left(\omega_{N}^{*}, \boldsymbol{u}_{N}^{*}\right)\right) \cdot\left(\theta_{N}, \boldsymbol{w}_{N}\right), \boldsymbol{v}_{N}\right\rangle \\
\quad=K_{N}\left(\tilde{\omega}_{N}-\omega_{N}^{*}, \boldsymbol{w}_{N} ; \boldsymbol{v}_{N}\right)+K_{N}\left(\theta_{N}, \tilde{\boldsymbol{u}}_{N}-\boldsymbol{u}_{N}^{*} ; \boldsymbol{v}_{N}\right) .
\end{aligned}
$$

So combining (4.8) and Lemma 4.3 leads to the desired property.

Lemma 4.8. Assume that the data $\boldsymbol{f}$ belong to $H^{\sigma}(\Omega)^{2}, \sigma>1$. If Assumption 4.5 is satisfied, the following estimate holds

$$
\begin{aligned}
\left\|\left(\tilde{\omega}_{N}, \tilde{\boldsymbol{u}}_{N}\right)+\mathcal{S}_{N} G_{N}\left(\tilde{\omega}_{N}, \tilde{\boldsymbol{u}}_{N}\right)\right\|_{\mathcal{X}} & \\
& \leq c(\omega, \boldsymbol{u})\left(N^{-s}\|(\omega, \boldsymbol{u})\|_{H^{s+1}(\Omega) \times H^{s}(\Omega)^{2}}+N^{-\sigma}\|\boldsymbol{f}\|_{H^{\sigma}(\Omega)^{2}}\right),
\end{aligned}
$$

for a constant $c(\omega, \boldsymbol{u})$ only depending on the solution $(\omega, \boldsymbol{u})$.

Proof: From equation (4.4), we derive

$$
\begin{aligned}
\|\left(\tilde{\omega}_{N}, \tilde{\boldsymbol{u}}_{N}\right)+ & \mathcal{S}_{N} G_{N}\left(\tilde{\omega}_{N}, \tilde{\boldsymbol{u}}_{N}\right)\left\|_{\mathcal{X}} \leq\right\|\left(\omega-\tilde{\omega}_{N}, \boldsymbol{u}-\tilde{\boldsymbol{u}}_{N}\right)\left\|_{\mathcal{X}}+\right\|\left(\mathcal{S}-\mathcal{S}_{N}\right) G(\omega, \boldsymbol{u}) \|_{\mathcal{X}} \\
& +\left\|\mathcal{S}_{N}\left(G(\omega, \boldsymbol{u})-G\left(\tilde{\omega}_{N}, \tilde{\boldsymbol{u}}_{N}\right)\right)\right\|_{\mathcal{X}}+\left\|\mathcal{S}_{N}\left(G\left(\tilde{\omega}_{N}, \tilde{\boldsymbol{u}}_{N}\right)-G_{N}\left(\tilde{\omega}_{N}, \tilde{\boldsymbol{u}}_{N}\right)\right)\right\|_{\mathcal{X}} .
\end{aligned}
$$

The bound for the first term in the right-hand side obviously follows from (4.14). By combining estimate (4.9) and Assumption 4.5, we also derive

$$
\left\|\left(\mathcal{S}-\mathcal{S}_{N}\right) G(\omega, \boldsymbol{u})\right\|_{\mathcal{X}} \leq c N^{-s}\|(\omega, \boldsymbol{u})\|_{\left.H^{s+1} \Omega\right) \times H^{s}(\Omega)^{2}} .
$$

On the other hand, we have

$$
\begin{aligned}
K\left(\omega, \boldsymbol{u} ; \boldsymbol{v}_{N}\right)-K\left(\tilde{\omega}_{N}, \tilde{\boldsymbol{u}}_{N} ; \boldsymbol{v}_{N}\right) & \\
& =K\left(\omega-\tilde{\omega}_{N}, \boldsymbol{u} ; \boldsymbol{v}_{N}\right)+K\left(\omega, \boldsymbol{u}-\tilde{\boldsymbol{u}}_{N} ; \boldsymbol{v}_{N}\right)-K\left(\omega-\tilde{\omega}_{N}, \boldsymbol{u}-\tilde{\boldsymbol{u}}_{N} ; \boldsymbol{v}_{N}\right) .
\end{aligned}
$$

Moreover it follows from Assumption 4.5 that $(\omega, \boldsymbol{u})$ belongs to $L^{\infty}(\Omega) \times L^{q}(\Omega)^{2}$ for some $q>2$. So, combining (4.8) and (4.14) with Remark 4.2 and a modified version of it taking into account this further regularity yields, with obvious notation for $c(\omega, \boldsymbol{u})$,

$$
\left\|\mathcal{S}_{N}\left(G(\omega, \boldsymbol{u})-G\left(\tilde{\omega}_{N}, \tilde{\boldsymbol{u}}_{N}\right)\right)\right\|_{\mathcal{X}} \leq c(\omega, \boldsymbol{u}) N^{-s}\left(1+N^{-s}|\log N|^{\frac{1}{2}}\right)\|(\omega, \boldsymbol{u})\|_{\left.H^{s+1} \Omega\right) \times H^{s}(\Omega)^{2}} .
$$

Finally, it follows from the exactness property (3.6) and the choice of $\left(\tilde{\omega}_{N}, \tilde{\boldsymbol{u}}_{N}\right)$ that, for all $\boldsymbol{v}_{N}$ in $\mathbb{D}_{N}$, the quantities $K\left(\tilde{\omega}_{N}, \tilde{\boldsymbol{u}}_{N} ; \boldsymbol{v}_{N}\right)$ and $K_{N}\left(\tilde{\omega}_{N}, \tilde{\boldsymbol{u}}_{N} ; \boldsymbol{v}_{N}\right)$ coincide. Thus, if $\Pi_{N-1}$ denotes the orthogonal projection operator from $L^{2}(\Omega)$ onto the space of functions such that their restrictions to all $\Omega_{k}, 1 \leq k \leq K$, belong to $\mathbb{P}_{N-1}\left(\Omega_{k}\right)$, adding and subtracting the quantity $\Pi_{N-1} \boldsymbol{f}$ in the last term and using (4.8) and (3.7) lead to

$$
\left\|\mathcal{S}_{N}\left(G\left(\tilde{\omega}_{N}, \tilde{\boldsymbol{u}}_{N}\right)-G_{N}\left(\tilde{\omega}_{N}, \tilde{\boldsymbol{u}}_{N}\right)\right)\right\|_{\mathcal{X}} \leq c\left(\left\|\boldsymbol{f}-\Pi_{N-1} \boldsymbol{f}\right\|_{L^{2}(\Omega)^{2}}+\left\|\boldsymbol{f}-\mathcal{I}_{N} \boldsymbol{f}\right\|_{L^{2}(\Omega)^{2}}\right) .
$$

The standard approximation properties of the operators $\Pi_{N-1}$ and $\mathcal{I}_{N}[7$, Thms $7.1 \&$ 14.2] yield

$$
\left\|\mathcal{S}_{N}\left(G\left(\tilde{\omega}_{N}, \tilde{\boldsymbol{u}}_{N}\right)-G_{N}\left(\tilde{\omega}_{N}, \tilde{\boldsymbol{u}}_{N}\right)\right)\right\|_{\mathcal{X}} \leq c N^{-\sigma}\|\boldsymbol{f}\|_{H^{\sigma}(\Omega)^{2}} .
$$

The desired bound is then derived by combining the previous estimates. 
We are now in a position to prove the error estimate.

Theorem 4.9. Assume that the data $\boldsymbol{f}$ belong to $H^{\sigma}(\Omega)^{2}, \sigma>1$, and that the solution $(\omega, \boldsymbol{u}, p)$ of problem (2.6) satisfies Assumptions 4.4 and 4.5. Then, there exists an integer $N_{\diamond}$ and a constant $c_{\diamond}$ such that, for all $N \geq N_{\diamond}$, problem (3.10) has a unique solution $\left(\omega_{N}, \boldsymbol{u}_{N}, p_{N}\right)$ such that

$$
\left\|\omega-\omega_{N}\right\|_{H^{1}(\Omega)}+\left\|\boldsymbol{u}-\boldsymbol{u}_{N}\right\|_{H(\operatorname{div}, \Omega)} \leq c_{\diamond}|\log N|^{-\frac{1}{2}} .
$$

Moreover this solution satisfies the following error estimate

$$
\begin{aligned}
& \left\|\omega-\omega_{N}\right\|_{H^{1}(\Omega)}+\left\|\boldsymbol{u}-\boldsymbol{u}_{N}\right\|_{H(\operatorname{div}, \Omega)}+\left\|p-p_{N}\right\|_{L^{2}(\Omega)} \\
& \quad \leq c(\omega, \boldsymbol{u})\left(N^{-s}\left(\|\omega\|_{H^{s+1}(\Omega)}+\|\boldsymbol{u}\|_{H^{s}(\Omega)^{2}}+\|p\|_{H^{s}(\Omega)}\right)+N^{-\sigma}\|\boldsymbol{f}\|_{H^{\sigma}(\Omega)^{2}}\right),
\end{aligned}
$$

for a constant $c(\omega, \boldsymbol{u})$ only depending on the solution $(\omega, \boldsymbol{u})$.

Proof: Combining Lemmas 4.6 to 4.8 with the Brezzi-Rappaz-Raviart theorem [8] (see also [13, Chap. IV, Thm 3.1]) yields that, for $N$ large enough, problem (3.15) has a unique solution $\left(\omega_{N}, \boldsymbol{u}_{N}\right)$ which satisfies (4.20) and the first part of (4.21). Moreover, thanks to the discrete inf-sup condition (3.20), there exists a unique $p_{N}$ in $\mathbb{M}_{N}$ such that

$$
\forall \boldsymbol{v}_{N} \in \mathbb{D}_{N}, \quad b_{N}\left(\boldsymbol{v}_{N}, p_{N}\right)=\left(\boldsymbol{f}, \boldsymbol{v}_{N}\right)_{N}-a_{N}\left(\omega_{N}, \boldsymbol{u}_{N} ; \boldsymbol{v}_{N}\right)-K_{N}\left(\omega_{N}, \boldsymbol{u}_{N} ; \boldsymbol{v}_{N}\right),
$$

whence the existence and local uniqueness result. Moreover, we have for any $q_{N}$ in $\mathbb{M}_{N}$

$$
\begin{aligned}
b_{N}\left(\boldsymbol{v}_{N}, p_{N}-q_{N}\right)=b\left(\boldsymbol{v}_{N}, p-q_{N}\right)-\left\langle\boldsymbol{f}, \boldsymbol{v}_{N}\right\rangle+\left(\boldsymbol{f}, \boldsymbol{v}_{N}\right)_{N} \\
+a\left(\omega-\omega_{N}, \boldsymbol{u}-\boldsymbol{u}_{N} ; \boldsymbol{v}_{N}\right)+\left(a-a_{N}\right)\left(\omega_{N}, \boldsymbol{u}_{N} ; \boldsymbol{v}_{N}\right) \\
+K\left(\omega, \boldsymbol{u} ; \boldsymbol{v}_{N}\right)-K_{N}\left(\omega_{N}, \boldsymbol{u}_{N} ; \boldsymbol{v}_{N}\right),
\end{aligned}
$$

so that the estimate for $\left\|p-p_{N}\right\|_{L^{2}(\Omega)}$ follows from (3.20), a triangle inequality and the same arguments as in the proof of Lemma 4.8.

Estimate (4.21) is fully optimal and justifies both the choice of the discretization and the use of over-integration. 


\section{The iterative algorithm and its convergence.}

Applying Newton's method to problem (4.7) consists in solving iteratively the equation

$$
\begin{aligned}
\left(\omega_{N}^{\ell}, \boldsymbol{u}_{N}^{\ell}\right)= & \left(\omega_{N}^{\ell-1}, \boldsymbol{u}_{N}^{\ell-1}\right) \\
& -\left(\operatorname{Id}+\mathcal{S}_{N} D G_{N}\left(\omega_{N}^{\ell-1}, \boldsymbol{u}_{N}^{\ell-1}\right)\right)^{-1}\left(\left(\omega_{N}^{\ell-1}, \boldsymbol{u}_{N}^{\ell-1}\right)+\mathcal{S}_{N} G_{N}\left(\omega_{N}^{\ell-1}, \boldsymbol{u}_{N}^{\ell-1}\right)\right)
\end{aligned}
$$

Multiplying both sides of this equation by $\operatorname{Id}+\mathcal{S}_{N} D G_{N}\left(\omega_{N}^{\ell-1}, \boldsymbol{u}_{N}^{\ell-1}\right)$ and using the infsup condition (3.20), we observe that this equation can equivalently be written as follows: Being given an initial guess $\left(\omega_{N}^{0}, \boldsymbol{u}_{N}^{0}\right)$ in $\mathbb{C}_{N} \times \mathbb{D}_{N}$, we solve the following problem, for $\ell \geq 1$,

Find $\left(\omega_{N}^{\ell}, \boldsymbol{u}_{N}^{\ell}, p_{N}^{\ell}\right)$ in $\mathbb{C}_{\mathbb{N}} \times \mathbb{D}_{N} \times \mathbb{M}_{N}$ such that

$$
\begin{aligned}
& \forall \boldsymbol{v}_{N} \in \mathbb{D}_{N}, \quad a_{N}\left(\omega_{N}^{\ell}, \boldsymbol{u}_{N}^{\ell} ; \boldsymbol{v}_{N}\right)+K_{N}\left(\omega_{N}^{\ell-1}, \boldsymbol{u}_{N}^{\ell} ; \boldsymbol{v}_{N}\right)+K_{N}\left(\omega_{N}^{\ell}, \boldsymbol{u}_{N}^{\ell-1} ; \boldsymbol{v}_{N}\right) \\
& \quad-K_{N}\left(\omega_{N}^{\ell-1}, \boldsymbol{u}_{N}^{\ell-1} ; \boldsymbol{v}_{N}\right)+b_{N}\left(\boldsymbol{v}_{N}, p_{N}^{\ell}\right)=\left(\left(\boldsymbol{f}, \boldsymbol{v}_{N}\right)\right)_{N}, \\
& \forall q_{N} \in \mathbb{M}_{N}, \quad b_{N}\left(\boldsymbol{u}_{N}^{\ell}, q_{N}\right)=0, \\
& \forall \varphi_{N} \in \mathbb{C}_{N}, \quad c_{N}\left(\omega_{N}^{\ell}, \boldsymbol{u}_{N}^{\ell} ; \varphi_{N}\right)=0 .
\end{aligned}
$$

It is readily checked that, for each value of $\ell$, problem (5.2) results into a square linear system.

The convergence of this method can easily be derived from [8] (see [13, Chap. IV, Thm 6.5]) owing to Lemmas 4.6 and 4.7 .

Theorem 5.1. Assume that the solution ( $\omega, \boldsymbol{u}, p)$ of problem (2.6) satisfies Assumption 4.4. Then, there exists an integer $N_{*}$ and a constant $c_{*}$ such that, for all $N \geq N_{*}$ and for any initial guess $\left(\omega_{N}^{0}, \boldsymbol{u}_{N}^{0}\right)$ in $\mathbb{C}_{N} \times \mathbb{D}_{N}$ such that

$$
\left\|\omega-\omega_{N}^{0}\right\|_{H(\operatorname{curl}, \Omega)}+\left\|\boldsymbol{u}-\boldsymbol{u}_{N}^{0}\right\|_{H(\operatorname{div}, \Omega)} \leq c_{*}|\log N|^{-\frac{1}{2}},
$$

problem (5.2) for each $\ell \geq 1$ has a unique solution $\left(\omega_{N}^{\ell}, \boldsymbol{u}_{N}^{\ell}, p_{N}^{\ell}\right)$. Moreover the sequence $\left(\omega_{N}^{\ell}, \boldsymbol{u}_{N}^{\ell}, p_{N}^{\ell}\right)_{\ell}$ converges in a quadratic way, towards the unique solution $\left(\omega_{N}, \boldsymbol{u}_{N}, p_{N}\right)$ of problem (3.10) satisfying (4.20), in the sense that

$$
\left\|\left(\omega_{N}^{\ell}, \boldsymbol{u}_{N}^{\ell}\right)-\left(\omega_{N}, \boldsymbol{u}_{N}\right)\right\|_{\mathcal{X}} \leq c\left\|\left(\omega_{N}^{\ell-1}, \boldsymbol{u}_{N}^{\ell-1}\right)-\left(\omega_{N}, \boldsymbol{u}_{N}\right)\right\|_{\mathcal{X}}^{2} .
$$

As standard for Newton's method, the key point is to exhibit an initial guess $\left(\omega_{N}^{0}, \boldsymbol{u}_{N}^{0}\right)$ satisfying (5.3). In order to do that, we have decided to use a continuation method. For simplicity, we set $\lambda=\frac{1}{\nu}$ and define the modified bilinear form

$$
\tilde{a}_{N}\left(\omega_{N}^{\ell}, \boldsymbol{u}_{N}^{\ell} ; \boldsymbol{v}_{N}\right)=\frac{1}{\nu} a_{N}\left(\omega_{N}^{\ell}, \boldsymbol{u}_{N}^{\ell} ; \boldsymbol{v}_{N}\right)
$$

We also introduce a pseudo-pressure $\tilde{p}_{N}^{\ell}=\frac{1}{\nu} p_{N}^{\ell}$. Thus, problem (5.2) can equivalently be written as 
Find $\left(\omega_{N}^{\ell}, \boldsymbol{u}_{N}^{\ell}, \tilde{p}_{N}^{\ell}\right)$ in $\mathbb{C}_{\mathbb{N}} \times \mathbb{D}_{N} \times \mathbb{M}_{N}$ such that

$$
\begin{array}{lc}
\forall \boldsymbol{v}_{N} \in \mathbb{D}_{N}, \quad \tilde{a}_{N}\left(\omega_{N}^{\ell}, \boldsymbol{u}_{N}^{\ell} ; \boldsymbol{v}_{N}\right)+\lambda K_{N}\left(\omega_{N}^{\ell-1}, \boldsymbol{u}_{N}^{\ell} ; \boldsymbol{v}_{N}\right)+\lambda K_{N}\left(\omega_{N}^{\ell}, \boldsymbol{u}_{N}^{\ell-1} ; \boldsymbol{v}_{N}\right) \\
\quad-\lambda K_{N}\left(\omega_{N}^{\ell-1}, \boldsymbol{u}_{N}^{\ell-1} ; \boldsymbol{v}_{N}\right)+b_{N}\left(\boldsymbol{v}_{N}, \tilde{p}_{N}^{\ell}\right)=\lambda\left(\left(\boldsymbol{f}, \boldsymbol{v}_{N}\right)\right)_{N}, \\
\forall q_{N} \in \mathbb{M}_{N}, \quad b_{N}\left(\boldsymbol{u}_{N}^{\ell}, q_{N}\right)=0 \\
\forall \varphi_{N} \in \mathbb{C}_{N}, \quad c_{N}\left(\omega_{N}^{\ell}, \boldsymbol{u}_{N}^{\ell} ; \varphi_{N}\right)=0,
\end{array}
$$

Next, we fix a sample of parameters $\left(\lambda_{m}\right)_{0 \leq m \leq M}$ such that

$$
0=\lambda_{0}<\lambda_{1}<\cdots<\lambda_{M}=\lambda .
$$

We agree to denote by $\left(\omega_{N}\left(\lambda_{m}\right)^{\ell}, \boldsymbol{u}_{N}\left(\lambda_{m}\right)^{\ell}, \tilde{p}_{N}\left(\lambda_{m}\right)^{\ell}\right)$ the solution of problem (5.5) with $\lambda$ replaced by $\lambda_{m}$. We also fix an integer $L \geq 1$. Next, we use the following algorithm:

(i) Initial step: For $\lambda_{0}=0$, we observe that the solution $\left(\omega_{N}(0), \boldsymbol{u}_{N}(0), \tilde{p}_{N}(0)\right)$ of the Stokes problem is zero.

(ii) Iterative step: Assuming that $\left(\omega_{N}\left(\lambda_{m-1}\right)^{L}, \boldsymbol{u}_{N}\left(\lambda_{m-1}\right)^{L}\right)$ is known, we take the intermediate initial guess $\left(\omega_{N}\left(\lambda_{m}\right)^{0}, \boldsymbol{u}_{N}\left(\lambda_{m}\right)^{0}\right)$ equal to $\left(\omega_{N}\left(\lambda_{m-1}\right)^{L}, \boldsymbol{u}_{N}\left(\lambda_{m-1}\right)^{L}\right)$ and solve problem (5.5) with $\lambda$ equal to $\lambda_{m}$ for $\ell=1, \ldots, L$.

The iterative step is performed until $m=M-1$ and the initial guess $\left(\omega_{N}^{0}, \boldsymbol{u}_{N}^{0}\right)$ is taken equal to $\left(\omega_{N}\left(\lambda_{M-1}\right)^{L}, \boldsymbol{u}_{N}\left(\lambda_{M-1}\right)^{L}\right)$.

The mapping: $\lambda \mapsto\left(\omega_{N}(\lambda), \boldsymbol{u}_{N}(\lambda), p_{N}(\lambda)\right)$, where $\left(\omega_{N}(\lambda), \boldsymbol{u}_{N}(\lambda), p_{N}(\lambda)\right)$ is the solution of problem (3.10) with $\nu=\frac{1}{\lambda}$, is clearly Lipschitz-continuous on any bounded interval of $\mathbb{R}_{+}$. So it could be checked that, when $\max _{1 \leq m \leq M} \lambda_{m}-\lambda_{m-1}$ is small enough and for $L$ large enough, the previous algorithm provides an initial guess $\left(\omega_{N}^{0}, \boldsymbol{u}_{N}^{0}\right)$ satisfying (5.3) for one of the solutions $(\omega, \boldsymbol{u}, p)$ of problem (2.6). However, for the numerical experiments, we work with low values of $M$ and $L$, and also smaller values of $N$ than the final one. But, in any case, the convergence seems likely. 


\section{Some numerical experiments.}

Problem (5.2) is very similar to a discrete Stokes problem. So we refer to [2, §5] for a detailed description of its implementation. As also explained in this reference, the global system (which is not symmetric) is solved via a GMRES method with local preconditioners, so that it has not to be assembled.

We first check the convergence of the discretization in the case of Taylor-Green flow. The domain is the square $\Omega=]-1,1\left[^{2}\right.$ divided into two rectangles $\left.\Omega_{1}=\right]-1,0[\times]-1,1[$ and $\left.\Omega_{2}=\right] 0,1[\times]-1,1[$. The exact solution is given by

$$
\boldsymbol{u}(x, y)=\left(\begin{array}{c}
-\sin (\pi x) \cos (\pi y) \\
\cos (\pi x) \sin (\pi y)
\end{array}\right), \quad p(x, y)=\cos ^{2}(\pi x)+\cos ^{2}(\pi y),
$$

and the viscosity $\nu$ is taken equal to $10^{-2}$. Figure 1 presents the $\log$ of the three errors

$$
\left\|\omega-\omega_{N}\right\|_{H^{1}(\Omega)}, \quad\left\|\boldsymbol{u}-\boldsymbol{u}_{N}\right\|_{H(\operatorname{div}, \Omega)}, \quad\left\|p-p_{N}\right\|_{L^{2}(\Omega)},
$$

as a function of $N$, for $N$ varying from 5 to 20 , after $L=6$ Newton's iterations. When compared with finite element results for a similar test, see [9], these curves confirm the exponential accuracy of spectral element methods for smooth solutions.

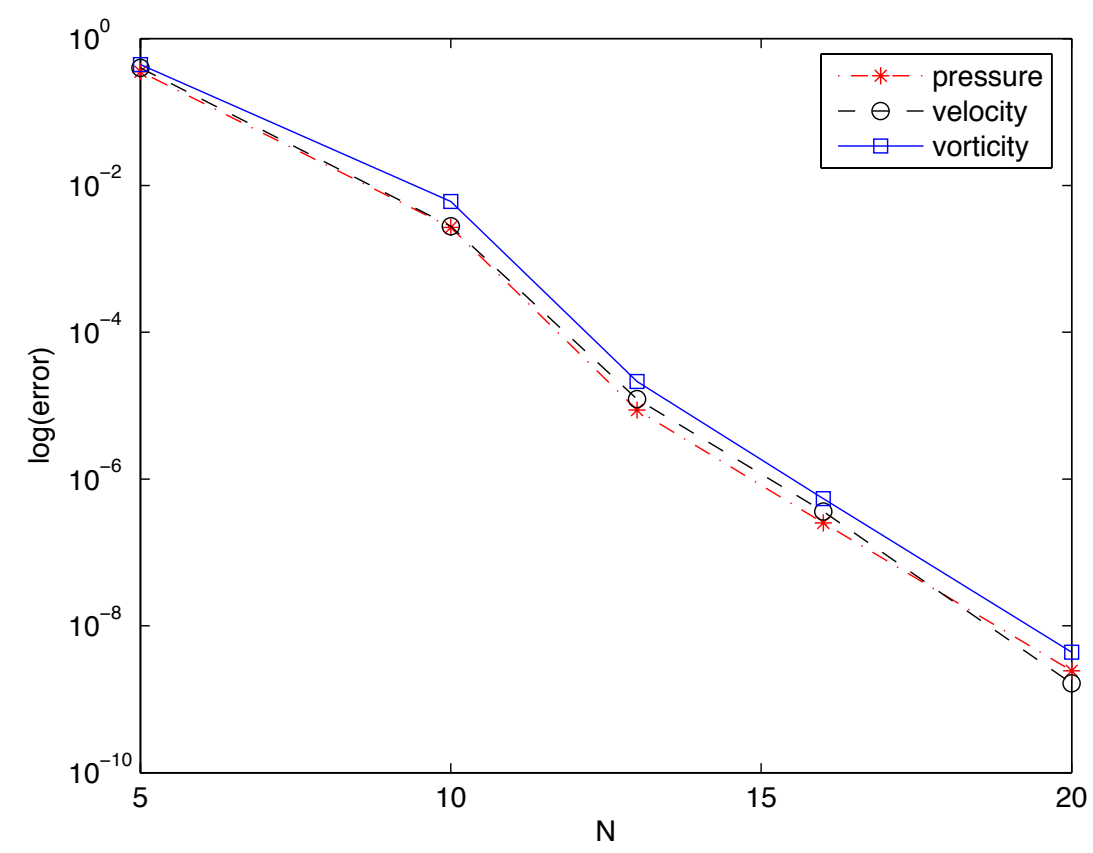

Figure 1: The error curves for a Taylor-Green flow

In the numerical experiments that we now present, the homogeneous boundary conditions on the velocity are replaced by

$$
\boldsymbol{u} \cdot \boldsymbol{n}=k \quad \text { on } \partial \Omega,
$$

where the datum $k$ satisfies the compatibility condition

$$
\int_{\partial \Omega} k(\tau) d \tau=0
$$


In the discrete case, this condition becomes

$$
\boldsymbol{u}_{N} \cdot \boldsymbol{n}=k_{N} \quad \text { on } \partial \Omega,
$$

where $k_{N}$ is defined in the following way: $\partial \Omega$ is the union of several segments $\Gamma_{\ell}$, and each $\Gamma_{\ell}$ is the union of one or several edges of the $\Omega_{k}$ that we denote by $\Gamma_{\ell, i}, 1 \leq i \leq I(\ell)$. Then each $k_{N \mid \Gamma_{\ell}}$ is defined as the image of $k_{\mid \Gamma_{\ell}}$ by the orthogonal projection operator from $L^{2}\left(\Gamma_{\ell}\right)$ onto the space

$$
\mathbb{T}\left(\Gamma_{\ell}\right)=\left\{g_{N} \in \mathscr{C}^{0}\left(\bar{\Gamma}_{\ell}\right) ; g_{N \mid \Gamma_{\ell, i}} \in \mathbb{P}_{N-1}\left(\Gamma_{\ell, i}\right), 1 \leq i \leq I(\ell)\right\} .
$$

A detailed analysis of the corresponding discrete problem is given in $[5, \S 5]$ for the Stokes problem. In particular, it is explained in [5, Rem. 5.4] that the function $k_{N}$ still satisfies condition (6.3), so that the corresponding discrete velocity $\boldsymbol{u}_{N}$ is exactly divergence-free. Of course, extending this analysis to the Navier-Stokes equations requires a Hopf lemma, which is rather technical (see [13, Chap. IV, Lemma 2.3] for instance). So, we prefer to skip this analysis for brevity.

We now consider the $L$-shaped domain $\Omega=]-1,1\left[{ }^{2} \backslash\left[0,1\left[^{2}\right.\right.\right.$, divided into three equal squares in an obvious way. We denote by $\Gamma_{1}$ the segment $\{-1\} \times[-1,1]$ and by $\Gamma_{2}$ the segment $\{1\} \times[-1,0]$, and we take the data $\boldsymbol{f}$ and $k$ defined by

$$
\begin{gathered}
\boldsymbol{f}(x, y)=(y, 0), \quad k(-1, y)=\left\{\begin{array}{ll}
-y(1-y) & \text { if } 0 \leq y \leq 1, \\
0 & \text { if }-1 \leq y \leq 0,
\end{array} \quad \text { on } \Gamma_{1},\right. \\
k(1, y)=-y(1+y) \quad \text { on } \Gamma_{2}, \quad k=0 \quad \text { on } \partial \Omega \backslash\left(\Gamma_{1} \cup \Gamma_{2}\right) .
\end{gathered}
$$

Note that the function $k$ satisfies (6.3) and moreover that its restriction to each $\Gamma_{\ell}$ belongs to the $\mathbb{T}\left(\Gamma_{\ell}\right)$ introduced in (6.5), so that $k_{N}$ is equal to $k$ on $\partial \Omega$. We take the viscosity $\nu$ equal to $10^{-2}$. Figure 2 presents, from top to bottom, the values of the vorticity, the two components of the velocity and the pressure for the discrete solution obtained with $N=23$. 

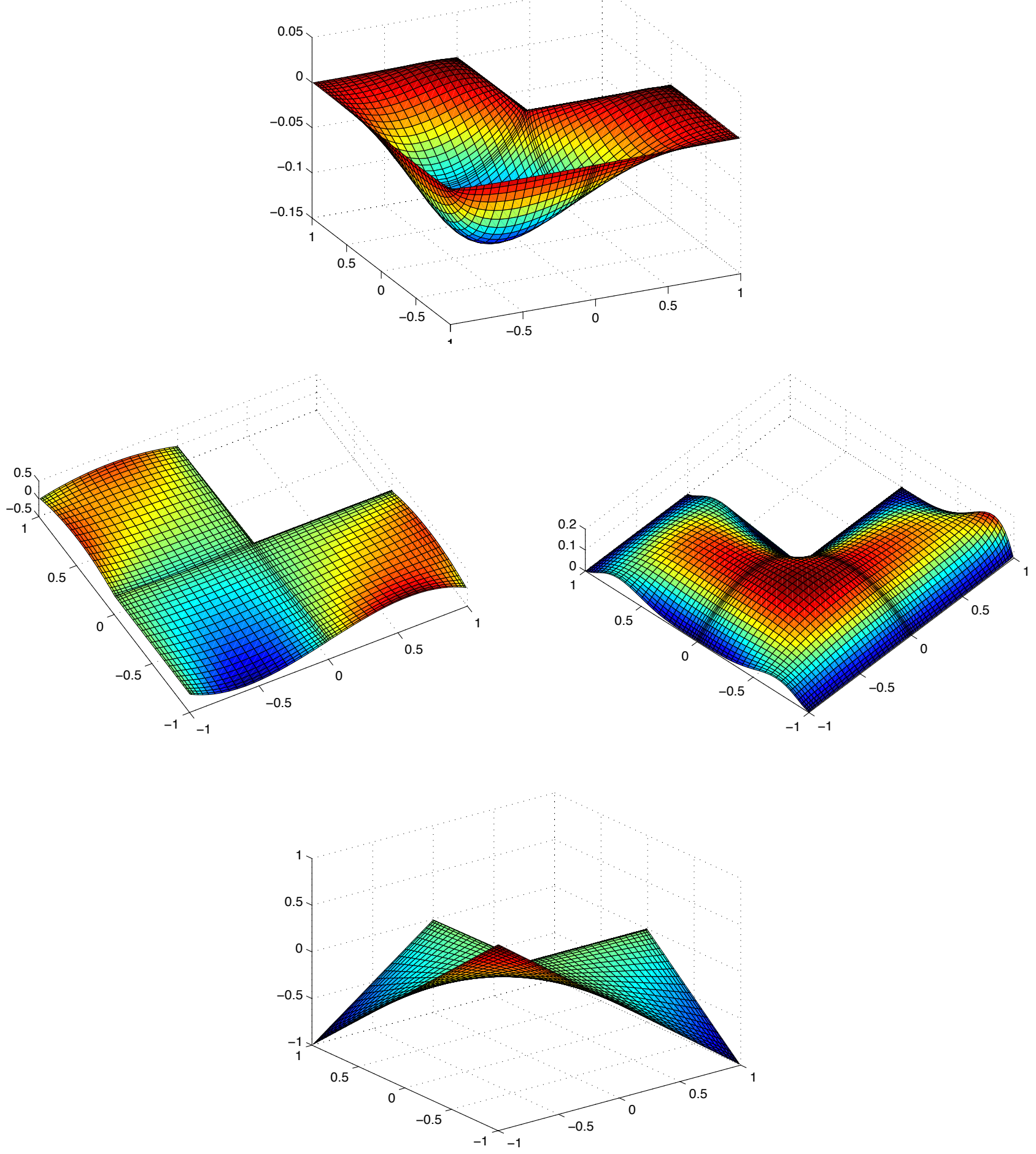

Figure 2: The isovalues of the vorticity, velocity and pressure for the $L$-shaped domain 
Finally, we consider the $U$-shaped domain $\Omega=]-2,2[\times]-2,1[\backslash[-1,1[2$, partitioned into two squares and three rectangles, see Figure 3: When turning counterclockwise,

$$
\begin{array}{r}
\left.\Omega_{1}=\right]-2,-1[\times]-1,1\left[, \quad \Omega_{2}=\right]-2,-1[\times]-2,-1\left[, \quad \Omega_{3}=\right]-1,1[\times]-2,-1[ \\
\left.\Omega_{4}=\right] 1,2[\times]-2,-1\left[, \quad \Omega_{5}=\right] 1,2[\times]-1,1[.
\end{array}
$$

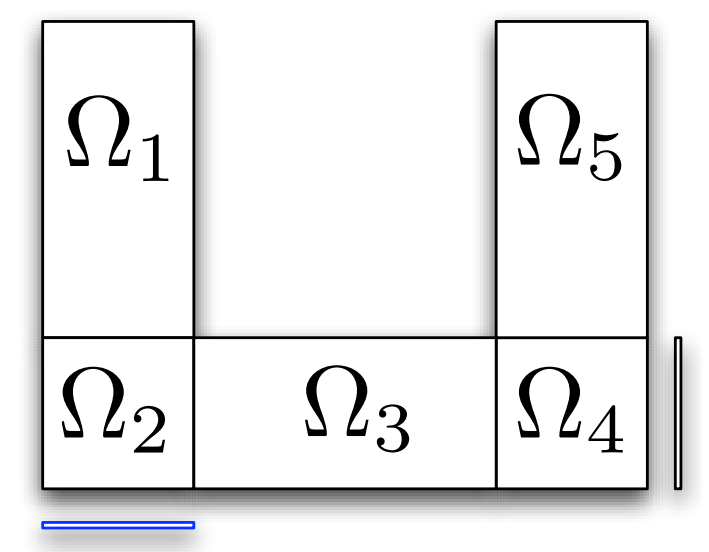

Figure 3: The $U$-shaped domain and its partition

The datum $\boldsymbol{f}$ is taken equal to zero; while the datum $k$ is given by

$$
k(x, y)= \begin{cases}-x \sin (\pi x) & \text { when }-2 \leq x \leq-1, y=-2, \\ y \sin (\pi y) & \text { when } x=2,-2 \leq y \leq-1, \\ 0 & \text { elsewhere. }\end{cases}
$$

So, it still satisfies (6.3) and vanishes on $\partial \Omega$ but in parts of $\partial \Omega_{2}$ and $\partial \Omega_{4}$ (as indicated in Figure 3). Still with $\nu$ equal to $10^{-2}$, Figure 4 presents, from top to bottom, the values of the vorticity, the two components of the velocity and the pressure for the discrete solution obtained with $N=23$. 

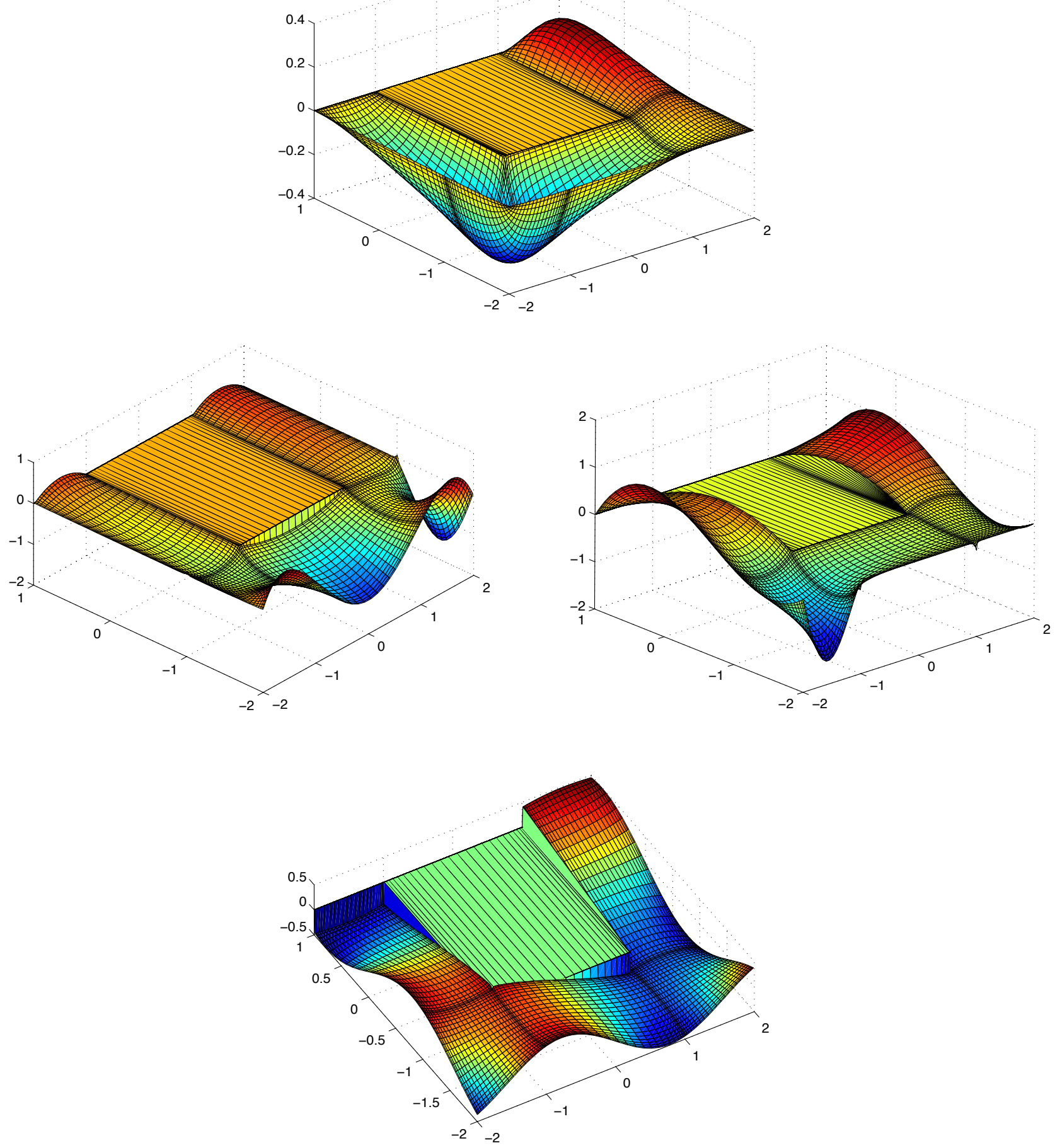

Figure 4: The isovalues of the vorticity, velocity and pressure for the $U$-shaped domain 


\section{References}

[1] M. Amara, D. Capatina-Papaghiuc, E. Chacón-Vera, D. Trujillo - Vorticity-velocity-pressure formulation for Navier-Stokes equations, Comput. Vis. Sci. 6 (2004), 47-52.

[2] K. Amoura, C. Bernardi, N. Chorfi - Spectral element discretization of the vorticity, velocity and pressure formulation of the Stokes problem, Modél. Math. et Anal. Numér. 40 (2006), $897-921$.

[3] C. Amrouche, C. Bernardi, M. Dauge, V. Girault - Vector potentials in three-dimensional nonsmooth domains, Math. Meth. in the Applied Sciences 21 (1998), 823-864.

[4] M. Azaïez, C. Bernardi, N. Chorfi - Spectral discretization of the vorticity, velocity and pressure formulation of the Navier-Stokes equations, Numer. Math. 104 (2006), 1-26.

[5] C. Bernardi, N. Chorfi - Spectral discretization of the vorticity, velocity and pressure formulation of the Stokes problem, SIAM J. Numer. Anal. 44 (2006), 826-850.

[6] C. Bernardi, V. Girault, P.-A. Raviart - Incompressible Viscous Fluids and their Finite Element Discretizations, in preparation.

[7] C. Bernardi, Y. Maday - Spectral Methods, in the Handbook of Numerical Analysis V, P.G. Ciarlet \& J.-L. Lions eds., North-Holland (1997), 209-485.

[8] F. Brezzi, J. Rappaz, P.-A. Raviart - Finite dimensional approximation of nonlinear problems, Part I: Branches of nonsingular solutions, Numer. Math. 36 (1980), 1-25.

[9] J.-P. Caltagirone, J. Breil — Sur une méthode de projection vectorielle pour la résolution des équations de Navier-Stokes, C. R. Acad. Sci. Paris 327 Série II b (1999), 1179-1184.

[10] M. Costabel - A remark on the regularity of solutions of Maxwell's equations on Lipschitz domains, Math. Meth. in Appl. Sc. 12 (1990), 365-368.

[11] F. Dubois - Vorticity-velocity-pressure formulation for the Stokes problem, Math. Meth. in the Applied Sciences 25 (2002), 1091-1119.

[12] F. Dubois, M. Salaün, S. Salmon - Vorticity-velocity-pressure and stream function-vorticity formulations for the Stokes problem, J. Math. Pures Appl. 82 (2003), 1395-1451.

[13] V. Girault, P.-A. Raviart - Finite Element Methods for Navier-Stokes Equations, Theory and Algorithms, Springer-Verlag (1986).

[14] Y. Maday, E.M. Rønquist - Optimal error analysis of spectral methods with emphasis on non-constant coefficients and deformed geometries, Comput. Methods in Applied Mech. and Engrg. 80 (1990), 91-115.

[15] J.-C. Nédélec — Mixed finite elements in $\mathbb{R}^{3}$, Numer. Math. 35 (1980), 315-341.

[16] R. Peyret, T.D. Taylor - Computational Methods for Fluid Flow, Springer-Verlag (1983).

[17] S. Salmon - Développement numérique de la formulation tourbillon-vitesse-pression pour le problème de Stokes, Thesis, Université Pierre et Marie Curie, Paris (1999). 